Título artículo / Títol article: Mechanistic Insight into the Lability of the Benzyloxycarbonyl (Z) Group inN-Protected Peptides under Mild Basic Conditions

Autores / Autors

Marta Tena Solsona, César A. Angulo Pachón, Beatriu Escuder, Juan F. Miravet Celades

Revista:

European Journal of Organic Chemistry, 2014, vol. 2014, no 16

Versión / Versió:

Post-print autor

Cita bibliográfica / Cita bibliogràfica (ISO 690):

url Repositori UJI:
TENA- SOLSONA, Marta, et al. Mechanistic Insight into the Lability of the Benzyloxycarbonyl (Z) Group in $\mathrm{N}$ - Protected Peptides under Mild Basic Conditions. European Journal of Organic Chemistry, 2014, vol. 2014, no 16, p. 3372-3378.

http://hdl.handle.net/10234/127265 


\title{
Mechanistic Insight into the Lability of Benzyloxycarbonyl (Z) Group in N- Protected Peptides under Mild Basic Conditions
}

\author{
Marta Tena-Solsona, César A. Angulo-Pachón, Beatriu Escuder* and Juan F. Miravet*
}

Keywords: Anchimeric assistance / neighbouring group participation / protecting groups / peptide chemistry / intramolecular catalysis

The unexpected lability of $\mathrm{Z}$ protecting group under mild basic conditions at room temperature is explained by a mechanism based on anchimeric assistance. It is found that the vicinal amide group stabilizes the tetrahedral intermediate formed after the nucleophilic addition of hydroxide to the carbonyl of the $\mathrm{Z}$ group. This effect operates in N-protected tripeptides and tetrapeptides but $\mathrm{Z}$ protected dipeptides are stable under the same conditions due to the blockage of the vicinal amide $\mathrm{NH}$ by intramolecular $\mathrm{H}$ bonding with terminal carboxylate moiety. [a] Departament de Química Inorgànica i Orgànica, Universitat Jaume I, Avda. Sos Baynat s/n, Castelló, 12071 Spain Fax: +34964728214

E-mail: miravet@uji.es; escuder@uji.es

Đomepage: http://supra.uji.es

Supporting information for this article is available on the WWW under http://dx.doi.org/10.1002/ejoc.xxxxxxxxx.

\section{Introduction}

Anchimeric assistance or neighbouring group participation constitutes a classic topic in organic chemistry and in particular in organic reaction mechanisms. Widespread examples of unusual reactivity associated to anchimeric assistance have been reported in the last decades and the relevance of this effect is still often highlighted nowadays in different areas of organic chemistry such as saccharides, ${ }^{1}$ synthesis of natural products, ${ }^{2}$ reactive intermediates, ${ }^{3}$ biochemical reactions, ${ }^{4}$ nucleotide chemistry ${ }^{5}$ or the reactivity of mustard reagents. ${ }^{6}$ Here we report on the lability of benzyloxycarbonyl ( $\mathrm{Z}$ thereafter) $\mathrm{N}$-protecting group in diluted aqueous $\mathrm{NaOH}$ at room temperature and on a mechanistic rationale for this behaviour based on anchimeric assistance of a vicinal amide group. The lability of $\mathrm{Z}$ group in cold, diluted sodium hydroxide solutions is unexpected if one considers that the literature on this protecting group in peptide and organic chemistry does not report such property. It is well known that the $\mathrm{Z}$ group can be cleaved by catalytic hydrogenolysis, acidolysis or, less commonly, by dissolving metal reduction. ${ }^{7,8}$ However, $\mathrm{Z}$ group is described to tolerate basic conditions ${ }^{9}$ and only a few reports are available of its removal under rather harsh conditions such as, refluxing with $\mathrm{Ba}(\mathrm{OH})_{2}$ in glyme/ $\mathrm{H}_{2} \mathrm{O}$ for $40 \mathrm{~h}$ or using $40 \%$ $\mathrm{NaOH}$ in $\mathrm{MeOH} / \mathrm{H}_{2} \mathrm{O} .^{10,11}$

\section{Results and Discussion}

Following our previous work on supramolecular hydrogels, ${ }^{12,13}$ three isomeric ionizable Z-protected tetrapeptides derived from aspartic acid and phenylalanine were prepared to be used as hydrogelators (Scheme 1). Unexpectedly, we noticed that upon dissolving the peptides in basic water $(0.1 \mathrm{M} \mathrm{NaOH})$ the $\mathrm{Z}$ groups were rapidly depleted from these molecules at room temperature. ${ }^{14}$ ${ }^{1} \mathrm{H}$ NMR spectra showed that the signal corresponding to the benzyl protons of the $\mathrm{Z}$ group progressively disappeared giving place to a new signal at at $4.57 \mathrm{ppm}$ which was assigned unambiguously to the benzylic protons of benzyl alcohol (Figure 1; notice that bottom spectrum is poorly resolved due to peptide aggregation. Well resolved spectra were obtained for smaller compounds shown in Table 1. See an example in SI.). A set of experiments was then designed to find out which structural factors of the peptides are important in this behavior and to propose a reasonable mechanism for this striking reactivity.

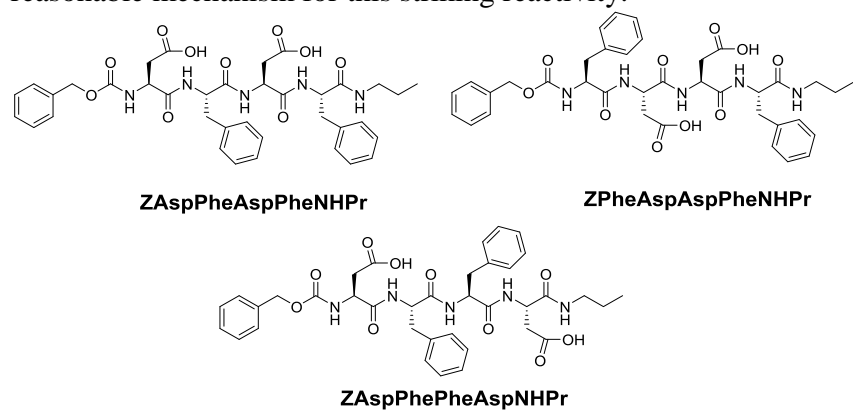

Scheme 1. Structure of the tetrapeptide derivatives studied.
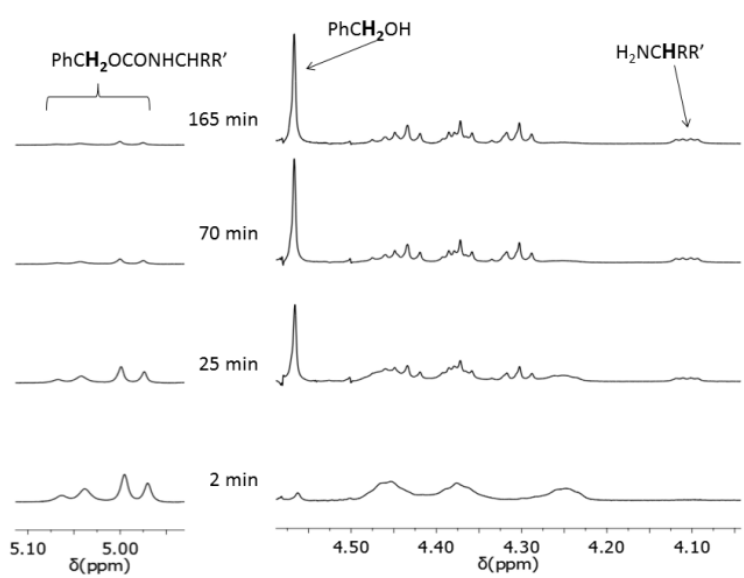

Figure 1. ${ }^{1} \mathrm{H}$ NMR spectra recorded for the basic hydrolysis of ZAspPhePheAspNHPr $(15 \mathrm{mM})$ in $\mathrm{D}_{2} \mathrm{O}([\mathrm{NaOH}]=0.1 \mathrm{M})$. 
First, a kinetic study was carried out in $\mathrm{D}_{2} \mathrm{O}$ and half-life times associated to pseudo first order kinetics were calculated for the tetrapeptides. Half-life times of 1-2 $\mathrm{h}$ were obtained in all the cases (entries 11-13, table 1) revealing a fast removal of the $Z$ group under the diluted basic conditions. We hypothesized that $\mathrm{Z}$ lability in those substrates could be explained considering that the tetrahedral intermediate formed upon nucleophilic attack of the hydroxide anion to the carbamate carbonyl is stabilized by intramolecular $\mathrm{H}$-bonding with the vicinal amide group as shown in Scheme 2 .
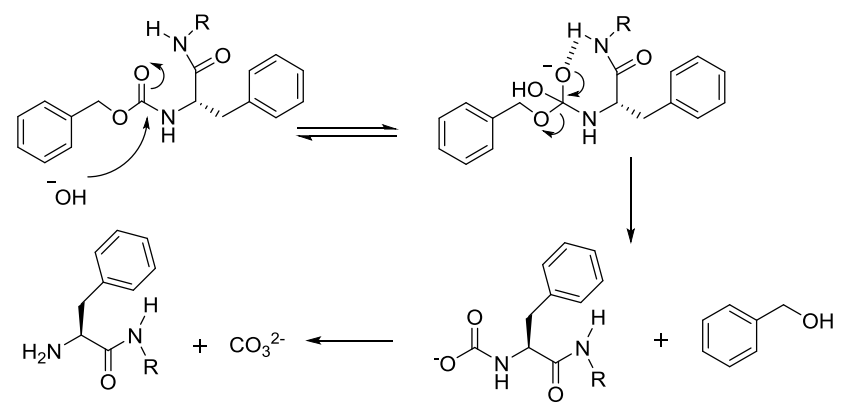

Scheme 2. Proposed mechanism for the basic hydrolysis of the carbamate group.

To prove the feasibility of this mechanism different Z-protected amino acids were studied (entries 1-8, Table 1). In $\mathrm{D}_{2} \mathrm{O}$ no hydrolysis was observed for Z-protected amino acids ( $\mathrm{ZValOH}$ and $\mathrm{ZPheOH}$ ) but hydrolysis took place readily when the carboxylic acid terminal unit was converted to amide as is the case of ZAspNHPr. Due to the low solubility of some of the compounds in water, we found very convenient to analyze the results obtained in methanol. As shown in the table, in this solvent no hydrolysis was observed for the carboxylic acid terminated amino acids (entries 12 ) but the Z-removal was rather fast when a terminal amide was present (entries 3-5). These results support the mechanism proposed in Scheme 2 which requires anchimeric assistance by a vicinal amide to stabilize the tetrahedral intermediate.

The hydrolysis of carbamates under basic conditions can take place via a BAc2 mechanism as depicted in scheme 2 or via an E1cB mechanism based on the deprotonation of the carbamate and formation of an intermediate isocyanate. ${ }^{15-18}$ To clarify this point, ZSarNHPr $(\mathrm{Sar}=$ sarcosine or $\mathrm{N}$-methylglycine $)$ was used as substrate. This amino acid derivative contains a N,N-disubstituted carbamate and therefore cannot participate in an E1cB mechanism due to the lack of a deprotonable NH unit. The fact that ZSarNHPr is also reacting in basic methanol at a rate even faster than the other amino acid derivatives (entry 6) agrees with a BAc2 mechanism taking place in this reaction. Furthermore, no reaction was observed for a $\mathrm{ZAspN}\left(\mathrm{CH}_{3}\right) \operatorname{Pr}$ or $\mathrm{ZPhe}\left(\mathrm{CH}_{3}\right) \mathrm{NPr}$ (entries 7 and 8), which are N-methylated analogues of ZAspNHPr and ZPheNHPr respectively, confirming the key role of the primary amide $\mathrm{NH}$ unit in the hydrolysis.

Next, to have results in a homopeptidic series the compounds ZGlyOH, ZGlyGlyOH and ZGlyGlyGlyOH were studied (entries 14-16, table 1). The assumption made was that the presence of the amide unit in a vicinal position to the carbamate unit would favor the hydrolytic Z-removal as was the case in the tetrapeptides reported above. So, under basic conditions no hydrolysis was expected to take place for ZGlyOH but Z-removal should occur for ZGlyGlyOH and ZGlyGlyGlyOH.
Table 1. Half-life times measured in basic media for different Z-protected amino acids and peptides at $30^{\circ} \mathrm{C}$

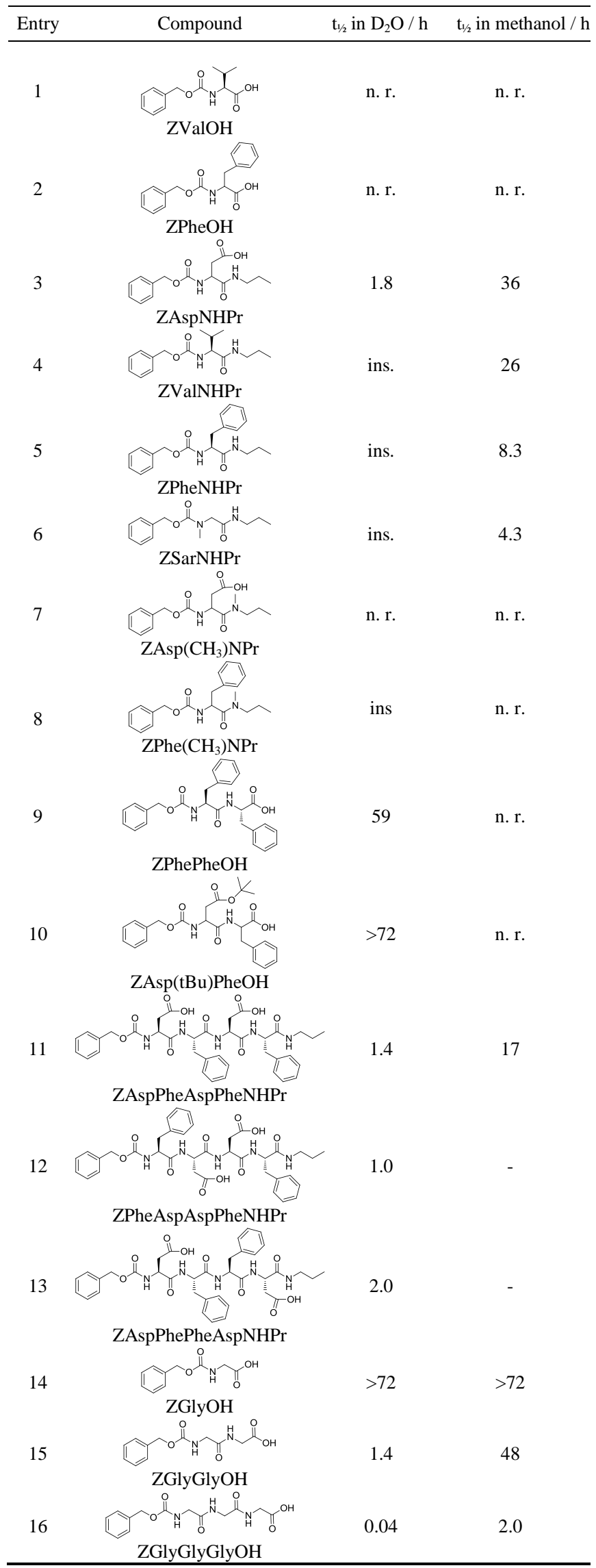

[a] n. r. = no reaction; ins. = insoluble. [Z-protected substrate $]=15 \mathrm{mM}$; $[\mathrm{NaOH}]=0.1 \mathrm{M}$. Half-life times were calculated considering a pseudo first order kinetics 
Sursprisingly, it was found that $\mathrm{Z}$ hydrolysis took place in all these compounds. Presumably the rather hydrophilic nature of Gly derivatives favors this reaction. However, very significant differences were found in the reactivity of these compounds. For the triglycine derivative in $\mathrm{D}_{2} \mathrm{O}$ a rather fast hydrolysis rate was measured with a half-life time of only $0.04 \mathrm{~h}$ but for $\mathrm{ZGlyOH}$ the reaction was much slower presenting a the half-life larger than $72 \mathrm{~h}$ It is noteworthy that the diglycine derivative ZGlyGlyOH reacted considerably slower that the corresponding tripeptide ZGlyGlyGlyOH, being this effect more pronounced in the methanolic basic solution with half-life times of $48 \mathrm{~h}$ and $2 \mathrm{~h}$ respectively. To confirm the hinted resistance to hydrolysis of the $\mathrm{Z}$-protected dipeptides, two Z-dipeptides available in the laboratory, $\mathrm{ZPhePheOH}$ and $\mathrm{ZAsp}(\mathrm{tBu}) \mathrm{PheOH}$, were studied (entries 9 and 10, table 1). Noticeably, in basic methanolic solutions no hydrolysis was observed at all for these compounds and in water, only a quite slow hydrolysis process was detected with half-life times larger than $59 \mathrm{~h}$ for both dipeptides. This puzzling difference found for Zprotected tripeptides or tetrapeptides when compared to Zdipeptides can be explained considering the intramolecular hydrogen bond between the terminal carboxylate unit in the deprotonated peptides and the $\mathrm{NH}$ of the vicinal peptidic bond (figure 2B). It should be noted that this intramolecular bond blocks the assistance of the amide $\mathrm{NH}$ in the stabilization of the tetrahedral intermediate required for carbamate hydrolysis. Intramolecular $\mathrm{H}$-bonding between terminal carboxylate and vicinal amide in peptides has been reported previously and clearly demonstrated by the NMR shift experienced by the amide $\mathrm{NH}$ resonance. ${ }^{12}$ Interestingly carbamate hydrolysis is not blocked for ZAspNHPr (entry 3, Table 1) despite the fact that this compound presents a pendant carboxylic acid moietiy. In this case an intramolecular $\mathrm{H}$-bond between carboxylate and amide $\mathrm{NH}$ would form a 7-membered which is not thermodynamically as favorable as the case described in figure $2 \mathrm{~B}$ (5-membered ring).

Molecular models with computational chemistry (DFT-B3LYP/321 G) agree well with the experimental results described. Minimum energy models could be obtained with the negatively charged tetrahedral intermediate stabilized with intramolecular $\mathrm{H}$ bonding from the adjacent amide bond (figure 2A). Furthermore, computational models also point out to the formation of a stable intramolecular $\mathrm{H}$-bond between terminal carboxylate unit and vicinal peptide (figure 2B).

Finally, due the widespread use of the related protecting group BOC (tertbutoxycarbonyl) the stability of BOCPheNHPr in the presence of $0.1 \mathrm{M} \mathrm{NaOH}$ in $\mathrm{MeOH}$ was also assesed. No hydrolysis was observed after $24 \mathrm{~h}$ in difference to ZPheNHPr (entry 5, Table 1). The resistance of BOCPheNHPr towards hydrolysis is in accordance to the well known resistance of tertbutyl esters to basic hydrolysis as a result of the steric hindrance provided by the tertbutyl unit.

\section{Conclusions}

In summary, anchimeric assistance by intramolecular H-bonding affords the stabilization of the tetrahedral intermediate associated to carbamate hydrolysis in a related way to that observed in enzymatic catalysis, in particular to the so called oxyanion holes. ${ }^{19,20}$ A subtle interplay among different H-bonds is key for the observed reactivity: intramolecular $\mathrm{H}$-bond between carboxylate terminal group and the adjacent amide explains the hydrolytic stability of Z-dipeptides but switching to larger Zpeptides results in the emergence of a intramolecular catalytic mechanism responsible of the observed hydrolysis. The labile nature of $\mathrm{Z}$ group under diluted base and room temperature conditions provides with new, unreported, incompatibilities for this widely used protecting group but is not synthetically useful because of the associated epimerization.

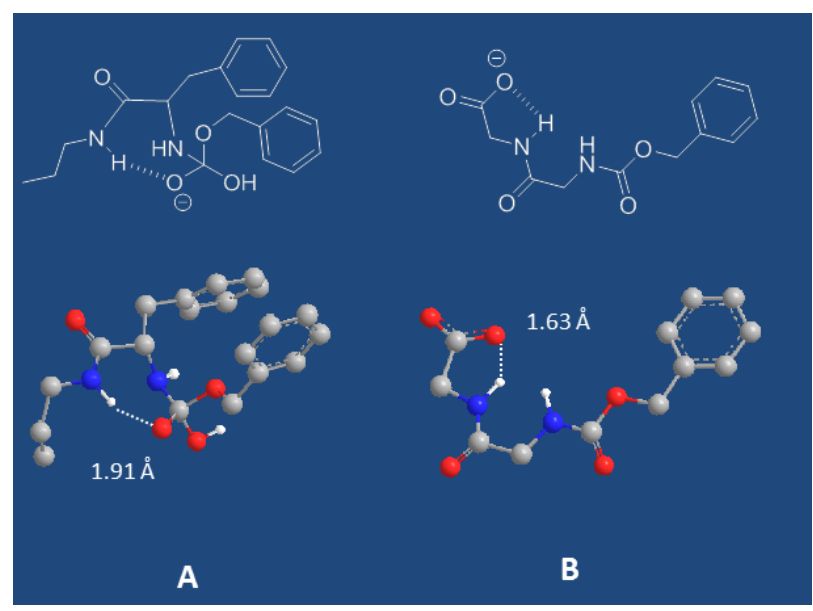

Figure 2. DFT-B3LYP/3-21 G energy minimized conformations (solvent model: PCM, H2O). The distances shown refer to the H-bond distances. A): Tetrahedral intermediate from the addition of hydroxide to ZPheNHPr. B): ZGlyGlyO-

\section{Experimental Section}

\subsection{General Considerations.}

NMR spectra were recorded at $500 \mathrm{MHz}, 300 \mathrm{MHz}\left({ }^{1} \mathrm{H}\right.$ NMR $)$ and $125 \mathrm{MHz}, 75 \mathrm{MHz}\left({ }^{13} \mathrm{C} \mathrm{NMR}\right)$ in different solvents at $30^{\circ} \mathrm{C}$ with the solvent signals as internal reference. Mass spectra were run in the electrospray (ESMS) mode. Optical rotations were measured at $25^{\circ} \mathrm{C}$. Reactions which required an inert atmosphere were carried out under dry $\mathrm{N}_{2}$ with flame-dried glassware. Commercial reagents were used as received. THF and DME were used as commercial available solvents.

\subsection{Synthesis.}

ZPheNHPr: The N-hydroxysuccinimide ester, ZPheOSu, (3.21 g, $8.7 \mathrm{mmol})$ was dissolved in DME $(50 \mathrm{~mL})$. 1-Aminopropane $(0.79$ $\mathrm{mL}, 9.6 \mathrm{mmol})$ dissolved in DME $(15 \mathrm{~mL})$ was added drop wise and the resulting solution was stirred at room temperature for 18 hours and then was warmed for 2 hours at $40-50^{\circ} \mathrm{C}$. The solvent was evaporated under vacuum. The resulting solid was dissolved in dichloromethane $(25 \mathrm{~mL})$ and washed three times with $\mathrm{HCl} 0.1 \mathrm{M}$ $(3 \times 25 \mathrm{~mL})$ and water $(3 \times 25 \mathrm{~mL})$. The organic phase was dried with anhydrous magnesium sulfate and the solvent was evaporated under vacuum. A white solid was obtained $(1.7 \mathrm{~g}, 4.99 \mathrm{mmol}$, 93\%). 1H NMR (500 MHz, $30^{\circ} \mathrm{C}$, d6-DMSO) $\delta 7.92(\mathrm{t}, \mathrm{J}=5.5 \mathrm{~Hz}$, $1 \mathrm{H}), 7.45(\mathrm{~d}, \mathrm{~J}=15.6 \mathrm{~Hz}, 1 \mathrm{H}), 7.38-7.10(\mathrm{~m}, 10 \mathrm{H}), 4.99-4.89$ (s, $2 \mathrm{H}), 4.21(\mathrm{~m}, 1 \mathrm{H}), 3.10-2.86(\mathrm{~m}, 3 \mathrm{H}), 2.77(\mathrm{dd}, J=13.6,10.1 \mathrm{~Hz}$, $1 \mathrm{H}), 1.45-1.30(\mathrm{~m}, 2 \mathrm{H}), 0.80(\mathrm{t}, J=7.4 \mathrm{~Hz}, 3 \mathrm{H}) .{ }^{13} \mathrm{C}$ NMR $(126$ $\mathrm{MHz}, 30^{\circ} \mathrm{C}, \mathrm{d}_{6}$-DMSO) $\delta 171.6,156.2,138.5,137.5,129.6,128.7$, $128.5,128.1,127.9,126.7,65.6,56.7,40.8,38.3,22.7,11.8$. (ESITOF, positive mode) $\mathrm{m} / \mathrm{z}$ exp $[\mathrm{M}+\mathrm{H}]^{+}$calcd for $\mathrm{C}_{20} \mathrm{H}_{25} \mathrm{~N}_{2} \mathrm{O}_{3}{ }^{+}$ 341.1865 ; found, $341.1860[\mathrm{M}+\mathrm{H}]^{+},(\Delta=1.5 \mathrm{ppm})$.

ZValNHPr: A similar procedure to that described for ZPheNHPr was used starting with ZValOSu (3.48 g, $10 \mathrm{mmol})$ and 1aminopropane $(0.90 \mathrm{~mL}, 11 \mathrm{mmol})$. A white solid was obtained (2.23 g, $7.62 \mathrm{mmol}, 85 \%)$. Compound ZValNHPr was previously described in literature and both ${ }^{1} \mathrm{H}$ and ${ }^{13} \mathrm{C}$ NMR spectra were in good agreement with the literature spectra. ${ }^{21}$ 
ZSarNHPr: A similar procedure to that described for ZPheNHPr was used starting with ZSarOSu (577 mg, $1.8 \mathrm{mmol})$ and 1aminopropane $(0.90 \mathrm{~mL}, 1.9 \mathrm{mmol})$. A white solid was obtained (0.36 g, $1.4 \mathrm{mmol}, 76 \%) .{ }^{1} \mathrm{H}$ NMR (500 MHz, $30{ }^{\circ} \mathrm{C}, \mathrm{d}_{6}$-DMSO) $\delta 7.86(\mathrm{~s}, 1 \mathrm{H}), 7.43-7.24(\mathrm{~m}, 5 \mathrm{H}), 5.05(\mathrm{ds}, 2 \mathrm{H}), 3.82(\mathrm{ds}, 2 \mathrm{H})$, $3.01(\mathrm{~m}, 2 \mathrm{H}), 2.87(\mathrm{ds}, 3 \mathrm{H}), 1.39(\mathrm{~m}, 2 \mathrm{H}), 0.92-0.72(\mathrm{dt}, J=7.4$ $\mathrm{Hz}, 3 \mathrm{H}) .{ }^{13} \mathrm{C}$ NMR $\left(75 \mathrm{MHz}, 30{ }^{\circ} \mathrm{C}, \mathrm{d}_{6}\right.$-DMSO) $\delta 168.9,168.4$, 156.4, 156.1, 137.4, 128.8, 128.7, 128.2, 128.4, 127.9, 127.5, 66.7, 66.5, 51.9, 51.6, 40.7, 36.4, 35.6, 22.8, 11.8. HRMS (ESI-TOF, positive mode) $\mathrm{m} / \mathrm{z} \exp [\mathrm{M}+\mathrm{H}]^{+}$calcd for $\mathrm{C}_{14} \mathrm{H}_{21} \mathrm{~N}_{2} \mathrm{O}_{3}{ }^{+}$ 265.1548 ; found, $265.1552[\mathrm{M}+\mathrm{H}]^{+},(\Delta=1.5 \mathrm{ppm})$.

ZPheN $\left(\mathrm{CH}_{3}\right)$ Pr: This compound was obtained by following the same synthesis described for the compound ZPheNHPr. The starting materials in this case were ZPheOsu (369 mg, $1 \mathrm{mmol}$ ) and $\mathrm{N}$-methylpropan-1-amine $(0.11 \mathrm{~mL}, 1.1 \mathrm{mmol})$. A transparent oil was obtained $(0.27 \mathrm{~g}, 0.76 \mathrm{mmol}, 76 \%)$. ${ }^{1} \mathrm{H}$ NMR $(300 \mathrm{MHz}, 30$ ${ }^{\circ} \mathrm{C}, \mathrm{d}_{6}$-DMSO) $\delta 7.63(\mathrm{dd}, J=8.3 \mathrm{~Hz}, 1 \mathrm{H}), 7.40-7.05(\mathrm{~m}, 10 \mathrm{H})$, $5.05-4.78(\mathrm{~m}, 2 \mathrm{H}), 4.59(\mathrm{~m}, 1 \mathrm{H}), 3.10-3.30(\mathrm{~m}, 4 \mathrm{H}), 2.95-2.62$ $(\mathrm{m}, 5 \mathrm{H}), 1.61-1.22(\mathrm{~m}, 2 \mathrm{H}), 0.74(\mathrm{dt}, J=7.6 \mathrm{~Hz}, 3 \mathrm{H}) .{ }^{13} \mathrm{C} \mathrm{NMR}$ (75 MHz, $30{ }^{\circ} \mathrm{C}, \mathrm{d}_{6}$-DMSO) $\delta 171.3,156.1,138.2,138.0,137.48$, $129.7,129.7,128.7,128.7,128.5,128.1,127.9,126.8,65.7,52.7$, $52.6,50.8,49.3,38.2,37.7,35.1,33.8,21.6,20.2,11.5,11.3$. HRMS (ESI-TOF, positive mode) $\mathrm{m} / \mathrm{z}$ exp $[\mathrm{M}+\mathrm{H}]^{+}$calcd for $\mathrm{C}_{2} 1 \mathrm{H}_{27} \mathrm{~N}_{2} \mathrm{O}_{3}{ }^{+} 355.2022$; found, $355.2022[\mathrm{M}+\mathrm{H}]^{+},(\Delta=0.0 \mathrm{ppm})$

ZAspNHPr: The reaction coupling between the Nhydroxysuccinimidyl ester, ZAsp(tBu)OSu (1.35 g, $3.2 \mathrm{mmol})$, and the corresponding amine $(3.5 \mathrm{mmol})$ was carried out in a similar way described above for ZPheNHPr. After that, an extra deprotection step was needed. Removal of $\mathrm{tBu}$ protecting group was carried based on the procedure reported in the literature.22 A solution t-butyl ester ZAsp(tBu)NHPr (1.09 $\mathrm{g}, 3.0 \mathrm{mmol})$ in a mixture of TFA (12 mL) (trifluroacetic), dichloromethane $(12 \mathrm{~mL})$ and water $(0.9 \mathrm{~mL})$ was stirred for $40 \mathrm{~min}$ at room temperature. Dichloromethane and TFA were evaporated under reduced pressure and the residue was co-distilled three times with eter. The obtained product was dissolved in $20 \mathrm{~mL}$ of dichloromethane and washed with $\mathrm{HCl} 0.1 \mathrm{M}(3 \times 25 \mathrm{ml})$ and water $(3 \times 25 \mathrm{ml})$. The organic phase was dried with magnesium sulfate anhydrous and the solvent was evaporated under vacuum. A white solid was obtained $(0.83 \mathrm{~g}, 2.69 \mathrm{mmol}$, Global Yield $=85 \%)$. 1H NMR $(500 \mathrm{MHz}, 30$ ${ }^{\circ} \mathrm{C}$, d6-DMSO) $\delta 7.81(\mathrm{t}, \mathrm{J}=5.1 \mathrm{~Hz}, 1 \mathrm{H}), 7.46(\mathrm{~d}, \mathrm{~J}=8.2 \mathrm{~Hz}, 1 \mathrm{H})$, $7.41-7.22(\mathrm{~m}, 5 \mathrm{H}), 5.10-4.97(\mathrm{~m}, 2 \mathrm{H}), 4.37-4.26(\mathrm{~m}, 1 \mathrm{H}), 3.08$ $-2.90(\mathrm{~m}, 2 \mathrm{H}), 2.71-2.56(\mathrm{~m}, 1 \mathrm{H}), 2.56-2.42(\mathrm{~m}, 1 \mathrm{H}), 1.44-$ $1.33(\mathrm{~m}, 2 \mathrm{H}), 0.81(\mathrm{t}, \mathrm{J}=7.4 \mathrm{~Hz}, 3 \mathrm{H}) .13 \mathrm{C} \mathrm{NMR}\left(126 \mathrm{MHz}, 30^{\circ} \mathrm{C}\right.$, d6-DMSO) $\delta 172.2,170.9,156.2,137.4,128.8,128.2,128.1,65.9$, 52.0, 40.9, 37.0, 22.7, 11.7. (ESI-TOF, negative mode) $\mathrm{m} / \mathrm{z}$ exp [M -H]- calcd for C15H19N2O5- 307.1294; found, 307.1295 [M -H]$(\Delta=0.3 \mathrm{ppm})$.

ZAspN $\left(\mathbf{C H}_{3}\right)$ Pr: A similar procedure to that described for ZAspNHPr was used. In this case the reaction coupling was between ZAsp(tBu)OSu (1.35 g, $3.2 \mathrm{mmol})$ and N-methylpropan1 -amine $(0.36 \mathrm{~mL}, 3.5 \mathrm{mmol})$. The deprotection with TFA and $\mathrm{CH}_{2} \mathrm{Cl}_{2}$ produced a transparent oil $(0.73 \mathrm{~g}, 2.26 \mathrm{mmol}$, Global Yield $=71 \%) .1 \mathrm{H} \mathrm{NMR}\left(300 \mathrm{MHz}, 30^{\circ} \mathrm{C}\right.$, d6-DMSO $) \delta 7.69(\mathrm{dd}$, $\mathrm{J}=11.9,9.1 \mathrm{~Hz}, 1 \mathrm{H}), 7.43-7.17(\mathrm{~m}, 5 \mathrm{H}), 5.03(\mathrm{~s}, 2 \mathrm{H}), 4.72(\mathrm{~m}$, $1 \mathrm{H}), 3.27(\mathrm{~m}, 2 \mathrm{H}), 3.00-2.77(\mathrm{ds}, 3 \mathrm{H}), 2.76-2.20(\mathrm{~m}, 2 \mathrm{H}), 1.47$ $(\mathrm{dt}, J=29.1,14.1,7.1 \mathrm{~Hz}, 13 \mathrm{H}), 0.78(\mathrm{dt}, J 7.4 \mathrm{~Hz}, 3 \mathrm{H}) .{ }^{13} \mathrm{C}$ NMR (75 MHz, $30^{\circ} \mathrm{C}, \mathrm{d}_{6}$-DMSO) $\delta 172.2,170.5,170.2,156.0,156.0$, $137.4,137.3,128.8,128.2,128.1,128.1,66.0,66.0,55.3,50.7$, 49.3, 49.0, 48.0, 37.3, 36.9, 35.0, 33.7, 21.5, 20.2, 11.4, 11.3. (ESITOF, negative mode) $\mathrm{m} / \mathrm{z}$ exp $[\mathrm{M}-\mathrm{H}]^{-}$calcd for $\mathrm{C}_{16} \mathrm{H}_{21} \mathrm{~N}_{2} \mathrm{O}_{5}$ 321.1450 ; found, $321.1451[\mathrm{M}-\mathrm{H}]^{-},(\Delta=0.3 \mathrm{ppm})$.

ZPhePheOH: In a first step the corresponding methyl ester of the dipeptide was obtained following a described procedure. ${ }^{23}$ The L-
Phenylalanine methyl ester hydrochloride (518 mg, $2.4 \mathrm{mmol}$ ) was dissolved in $30 \mathrm{~mL}$ of DME (dimethoxyethane). Then triethylamine were added $(0.36 \mathrm{~mL}, 2.6 \mathrm{mmol})$ and immediately a white precipitate appeared. A solution of ZPheOSu $(887 \mathrm{mg}, 2.4$ $\mathrm{mmol})$ in DME $(30 \mathrm{~mL})$ were added drop wise and the reaction mixture was stirred at room temperature 2 hours, filtered and evaporated. The residue was dissolved in dichloromethane $(25 \mathrm{~mL})$ and washed four times with water $(25 \mathrm{~mL})$. The organic phase was dried with magnesium sulfate anhydrous and the solvent evaporated under vacuum.

Then, following a reported procedure,${ }^{24}$ the corresponding methyl ester $(875 \mathrm{mg}, 1.9 \mathrm{mmol})$ was dissolved in THF $(20 \mathrm{~mL})$ and cooled at $0{ }^{\circ} \mathrm{C}$. A solution of lithium hydroxide $(62.4 \mathrm{mg}, 2.6$ $\mathrm{mmol})$ in water $(10 \mathrm{~mL})$ was added drop wise. The reaction mixture was stirred for 0.5 hour. Then this mixture was washed with ether $(20 \mathrm{~mL})$. The aqueous layer was acidified with with 1 $\mathrm{M} \mathrm{HCl}$ to adjust to $\mathrm{pH} 2$ and extracted with ether $(3 \mathrm{x} 30 \mathrm{~mL})$. The organic extracts were combined, dried with magnesium sulfate anhydrous powder and evaporated.

A white solid was obtain $(0.92 \mathrm{~g}, 2.06 \mathrm{mmol}$, Global Yield $82 \%)$. ${ }^{1} \mathrm{H}$ NMR $\left(300 \mathrm{MHz}, 30{ }^{\circ} \mathrm{C}, \mathrm{d}_{6}\right.$-DMSO) $\delta 8.23(\mathrm{~d}, J=7.8 \mathrm{~Hz}, 1 \mathrm{H})$, $7.42(\mathrm{~d}, J=8.8 \mathrm{~Hz}, 1 \mathrm{H}), 7.36-6.94(\mathrm{~m}, 15 \mathrm{H}), 5.03-4.81(\mathrm{~s}, 2 \mathrm{H})$, $4.57-4.38(\mathrm{~m}, 1 \mathrm{H}), 4.29(\mathrm{~m}, 1 \mathrm{H}), 3.43(\mathrm{dd}, J=41.4,34.4 \mathrm{~Hz}$ $2 \mathrm{H}), 3.15-2.81(\mathrm{~m}, 3 \mathrm{H}), 2.70(\mathrm{dd}, J=13.7,10.9 \mathrm{~Hz}, 1 \mathrm{H}) .{ }^{13} \mathrm{C}$ NMR $\left(75 \mathrm{MHz}, 30{ }^{\circ} \mathrm{C}, \mathrm{d}_{6}\right.$-DMSO) $\delta 173.2,172.0,156.2,138.5$, 137.8, 137.4, 129.6, 128.7, 128.6, 128.4, 128.1, 127.8, 127.2, 126.9, 126.7, 65.6, 56.4, 53.9, 37.9, 37.2. (ESI-TOF, positive mode) $\mathrm{m} / \mathrm{z}$ $\exp [\mathrm{M}+\mathrm{Na}]^{+}$calcd for $\mathrm{C}_{26} \mathrm{H}_{26} \mathrm{~N}_{2} \mathrm{NaO}_{5}{ }^{+} 469.1739$; found, $369.1737[\mathrm{M}+\mathrm{Na}]^{+},(\Delta=0.4 \mathrm{ppm})$.

ZAsp(tBu)PheOH: A similar procedure to that described for $\mathrm{ZPhePheOH}$ was used. The starting materials in this case were LPhenylalanine methyl ester hydrochloride $(518 \mathrm{mg}, 2.4 \mathrm{mmol})$ triethylamine $(0.36 \mathrm{~mL}, 2.6 \mathrm{mmol})$ and $\mathrm{ZAsp}(\mathrm{tBu}) \mathrm{OSu}(1.00 \mathrm{~g}, 2.4$ $\mathrm{mmol})$. A white solid was obtained $(0.89 \mathrm{~g}, 1.89 \mathrm{mmol}$, Global Yield $75 \%) .{ }^{1} \mathrm{H}$ NMR $\left(300 \mathrm{MHz}, 30{ }^{\circ} \mathrm{C}, \mathrm{d}_{6}\right.$-DMSO) $\delta 7.98(\mathrm{~d}, J=$ $7.4 \mathrm{~Hz}, 1 \mathrm{H}), 7.51(\mathrm{~d}, J=8.4 \mathrm{~Hz}, 1 \mathrm{H}), 7.42-7.12(\mathrm{~m}, 10 \mathrm{H}), 5.12$ $4.91(\mathrm{~m}, 2 \mathrm{H}), 4.49-4.25(\mathrm{~m}, 2 \mathrm{H}), 3.07-2.85(\mathrm{~m}, 2 \mathrm{H}), 2.71-2.30$ $(\mathrm{m}, 2 \mathrm{H}), 1.36(\mathrm{~s}, 9 \mathrm{H}) .{ }^{13} \mathrm{C} \mathrm{NMR}\left(75 \mathrm{MHz}, 30^{\circ} \mathrm{C}, \mathrm{d}_{6}\right.$-DMSO) $\delta$ 173.0, 171.3, 171.0, 169.6, 156.1, 137.7, 137.3, 129.6, 128.8, 128.6, $128.2,128.1,126.9,80.6,65.9,53.9,51.8,38.0,37.0,28.1$. (ESITOF, positive mode) $\mathrm{m} / \mathrm{z} \exp [\mathrm{M}+\mathrm{Na}]^{+}$calcd for $\mathrm{C}_{25} \mathrm{H}_{30} \mathrm{~N}_{2} \mathrm{NaO}_{7}{ }^{+}$ 493.1951 ; found, $493.1947[\mathrm{M}+\mathrm{Na}]^{+},(\Delta=0.8 \mathrm{ppm})$.

The following peptides were obtained using conventional solution peptide chemistry. See supporting information for synthetic scheme. General procedures are shown below.

General procedure for peptide coupling. The $\mathrm{N}$ hydroxysuccinimide ester of the Z-protected amino acid (8.7 $\mathrm{mmol})$ was dissolved in DME $(50 \mathrm{~mL})$. Then corresponding peptide or amino acid with the C-terminal group as propylamide $(8.7 \mathrm{mmol})$ was dissolved in DME $(15 \mathrm{~mL})$ and added drop wise. The resulting solution was stirred at room temperature for 18 hours and then was warmed for 2 hours at $40-50^{\circ} \mathrm{C}$. The solvent was evaporated under vacuum. The resulting solid was washed with sodium hydrogencarbonate saturated solution, $\mathrm{HCl} 0.1 \mathrm{M}$ aqueous solution and water. The final product was dried at $40^{\circ} \mathrm{C}$ under vacuum.

General procedure for N-benzyloxycarbonyl deprotection. The corresponding N-benzyloxycarbonyl protected peptide or amino acide derivative $(7.8 \mathrm{mmol})$ and catalytic amount of $\mathrm{Pd}$ over activated carbon $(5-10 \% \mathrm{w} / \mathrm{w})$ were placed in a two necked round bottom flask and suspended in $\mathrm{MeOH}(50 \mathrm{~mL})$. The system was purged to remove the air with $\mathrm{N}_{2}$ and connected to $\mathrm{H}_{2}$ atmosphere. 
The pasty grey suspension was stirred for several hours until it turned completely black (also checked with TLC, $\mathrm{MeOH}: \mathrm{CH}_{2} \mathrm{Cl}_{2}$ (2:8) and revealed with ninhydrin). The black suspension was filtered over celite and the solvent was evaporated under reduced pressure. The resulting oil was dried further in vacuum pump for 24 hours.

General procedure t-butyl ester group deprotection. A solution of tbutyl ester $(3.0 \mathrm{mmol})$ in a mixture of TFA $(12 \mathrm{~mL})$ (trifluroacetic), dichloromethane $(12 \mathrm{~mL})$ and water $(0.9 \mathrm{~mL})$ was stirred for 40 min at room temperature. Dichloromethane and TFA were evaporated under reduced pressure and the residue was co-distilled three times with eter. The solid was washed with water.

ZPheAspAspPheNHPr: A white solid was obtained (2.8 g, 3.9 mmol, Global Yield $51 \%){ }^{1} \mathrm{H}$ NMR $\left(500 \mathrm{MHz}, 30^{\circ} \mathrm{C}, \delta_{6}\right.$-DMSO) $\delta 8.40(\mathrm{~d}, J=7.5 \mathrm{~Hz}, 1 \mathrm{H}), 8.15(\mathrm{~d}, J=7.5 \mathrm{~Hz}, 1 \mathrm{H}), 7.77(\mathrm{~d}, J=8.2$ $\mathrm{Hz}, 1 \mathrm{H}), 7.62(\mathrm{t}, J=5.5 \mathrm{~Hz}, 1 \mathrm{H}), 7.46(\mathrm{~d}, J=8.5 \mathrm{~Hz}, 1 \mathrm{H}), 7.39-$ $6.96(\mathrm{~m}, 15 \mathrm{H}), 4.97-4.87(\mathrm{~m}, 2 \mathrm{H}), 4.58-4.54(\mathrm{~m}, 1 \mathrm{H}), 4.50-4.56$ $(\mathrm{m}, 1 \mathrm{H}), 4.38-4.34(\mathrm{~m}, 1 \mathrm{H}), 4.32-4.21(\mathrm{~m}, 1 \mathrm{H}), 3.08-2.88(\mathrm{~m}$, $4 \mathrm{H}), 2.86-2.81(\mathrm{~m}, 1 \mathrm{H}), 2.78-2.39(\mathrm{~m}, 6 \mathrm{H}), 1.43-1.27(\mathrm{~m}, 2 \mathrm{H})$, $0.76(\mathrm{t}, J=7.4 \mathrm{~Hz}, 3 \mathrm{H}) .{ }^{13} \mathrm{C}$ NMR $\left(75 \mathrm{MHz}, 30{ }^{\circ} \mathrm{C}, \mathrm{d}_{6}\right.$-DMSO) $\delta$ $172.3,172.2,172.2,171.2,170.6,170.4,156.3,138.5,138.2,137.4$ $129.7,129.5,128.7,128.5,128.4,128.1,127.9,126.7,65.7,56.4$, 54.7, 50.2, 50.0, 37.8, 36.5, 36.2, 22.6, 11.7. (ESI-TOF, negative mode) $\mathrm{m} / \mathrm{z}$ exp $[\mathrm{M}-\mathrm{H}]^{-}$calcd for $\mathrm{C}_{37} \mathrm{H}_{42} \mathrm{~N}_{5} \mathrm{O}_{10}{ }^{-} 716.2932$; found, $716.2924[\mathrm{M}-\mathrm{H}]^{-},(\Delta=1.1 \mathrm{ppm})$.

ZAspPhePheAspNHPr: A White solid was obtained (1.5 g, 2.09 mmol, Global Yield $45 \%)$. ${ }^{1} \mathrm{H}$ NMR $\left(300 \mathrm{MHz}, 30^{\circ} \mathrm{C}, \mathrm{d}_{6}\right.$-DMSO) $\delta 8.16(\mathrm{dd}, J=7.4 \mathrm{~Hz}, 2 \mathrm{H}), 7.78(\mathrm{~d}, J=7.6 \mathrm{~Hz}, 1 \mathrm{H}), 7.50(\mathrm{~m}, 2 \mathrm{H})$, 7.36-7.10 (m, 15H), $5.12-4.87(\mathrm{~m}, 2 \mathrm{H}), 4.62-4.16(\mathrm{~m}, 4 \mathrm{H}), 3.10$ $-2.16(\mathrm{~m}, 11 \mathrm{H}), 1.51-1.27(\mathrm{~m}, 2 \mathrm{H}), 0.80(\mathrm{t}, J=7.4 \mathrm{~Hz}, 3 \mathrm{H}) .{ }^{13} \mathrm{C}$ NMR $\left(75 \mathrm{MHz}, 30^{\circ} \mathrm{C}, \mathrm{d}_{6}\right.$-DMSO) $\delta 172.2,172.2,171.2,171.1$, $171.1,170.3,156.2,137.9,137.8,137.3,129.7,129.6,128.8,128.5$ 128.4, 128.2, 128.1, 126.8, 126.6, 66.0, 54.5, 54.1, 51.8, 50.1, 40.9, $37.8,36.7,36.5,22.6,11.7$. (ESI-TOF, negative mode) $\mathrm{m} / \mathrm{z}$ exp [M $-\mathrm{H}]^{-}$calcd for $\mathrm{C}_{37} \mathrm{H}_{42} \mathrm{~N}_{5} \mathrm{O}_{10}{ }^{-} 716.2932$; found, $716.2926[\mathrm{M} \mathrm{-H}]^{-}$, $(\Delta=0.8 \mathrm{ppm})$

ZAspPheAspPheNHPr: A white solid was obtain (1.46 g, 2.03 mmol, Global Yield $52 \%) .{ }^{1} \mathrm{H}$ NMR $\left(300 \mathrm{MHz}, 30{ }^{\circ} \mathrm{C}, \mathrm{d}_{6}\right.$-DMSO) $\delta 8.28(\mathrm{~d}, J=7.2 \mathrm{~Hz}, 1 \mathrm{H}), 7.85(\mathrm{dd}, J=8.2 \mathrm{~Hz}, 2 \mathrm{H}), 7.70(\mathrm{t}, J=$ $5.8 \mathrm{~Hz}, 1 \mathrm{H}), 7.51(\mathrm{~d}, J=7.8 \mathrm{~Hz}, 1 \mathrm{H}), 7.42-7.03(\mathrm{~m}, 15 \mathrm{H}), 5.09-$ $4.91(\mathrm{~m}, 2 \mathrm{H}), 4.58-4.23(\mathrm{~m}, 4 \mathrm{H}), 2.48(\mathrm{~m}, 11 \mathrm{H}), 1.31(\mathrm{~m}, 2 \mathrm{H})$, $0.75(\mathrm{t}, J=7.4 \mathrm{~Hz}, 3 \mathrm{H}) \cdot{ }^{13} \mathrm{C}$ NMR $\left(75 \mathrm{MHz}, 30^{\circ} \mathrm{C}, \mathrm{d}_{6}-\mathrm{DMSO}\right) \delta$ 172.3, 172.2, 171.3, 171.2, 170.6, 170.5, 156.2, 138.1, 137.9, 137.3, 130.5, 129.7, 129.5, 128.8, 128.5, 128.4, 128.2, 128.1, 126.7, 66.0, 54.6, 54.2, 51.8, 50.1, 38.0, 37.9, 36.6, 36.4, 22.6, 11.7. (ESI-TOF, negative mode) $\mathrm{m} / \mathrm{z}$ exp $[\mathrm{M}-\mathrm{H}]^{-}$calcd for $\mathrm{C}_{37} \mathrm{H}_{42} \mathrm{~N}_{5} \mathrm{O}_{10}$ 716.2932 ; found, $716.2930[\mathrm{M}-\mathrm{H}]^{-},(\Delta=0.3 \mathrm{ppm})$.

ZGlyGlyOH: GlyGly (304 mg, $2.3 \mathrm{mmol}$ ) was dissolved in a $4 \mathrm{~N}$ aqueous solution of sodium hydroxide $(0.7 \mathrm{~mL})$. The solution was cooled at $0^{\circ} \mathrm{C}$ and a $4 \mathrm{~N}$ aqueous solution of sodium hydroxide (1.4 $\mathrm{mL})$ and benzyloxycarbonylchloride $(0.40 \mathrm{~mL}, 2.8 \mathrm{mmol})$ were added drop wise. Then the mixture was stirred overnight at $20^{\circ} \mathrm{C}$. The reaction mixture was washed with ether and the aqueous layer was cooled at $0{ }^{\circ} \mathrm{C}$. To the aqueous layer was added $5 \mathrm{M} \mathrm{HCl}$ to adjust its $\mathrm{pH}$ to 3 which was left standing overnight at a cool place. Resulting crystalline precipitates were collected by filtration, washed with cool water and dried. White crystals were obtain $(0.43$ g, $1.61 \mathrm{mmol}, 70 \%) .{ }^{1} \mathrm{H}$ NMR $\left(500 \mathrm{MHz}, 30{ }^{\circ} \mathrm{C}, \mathrm{d}_{6}\right.$-DMSO) $\delta$ $8.12(\mathrm{t}, J=5.5 \mathrm{~Hz}, 1 \mathrm{H}), 7.51-7.42(\mathrm{t}, J=6.2 \mathrm{~Hz} 1 \mathrm{H}), 7.41-7.25$ $(\mathrm{m}, 5 \mathrm{H}), 5.04(\mathrm{~s}, 2 \mathrm{H}), 3.76(\mathrm{t}, J=6.2 \mathrm{~Hz}, 2 \mathrm{H}), 3.68(\mathrm{~d}, J=6.4 \mathrm{~Hz}$ $2 \mathrm{H}) .{ }^{13} \mathrm{C}$ NMR $\left(126 \mathrm{MHz}, 30^{\circ} \mathrm{C}, \mathrm{d}_{6}\right.$-DMSO) $\delta 171.1,169.5,156.5$,
137.0, 128.4, 127.8, 127.7, 65.5, 43.4, 40.6. (ESI-TOF, negative mode) $\mathrm{m} / \mathrm{z} \exp [\mathrm{M}-\mathrm{H}]^{-}$calcd for $\mathrm{C}_{12} \mathrm{H}_{13} \mathrm{~N}_{2} \mathrm{O}_{5}{ }^{-} 265.0824$; found, $265.0819[\mathrm{M}-\mathrm{H}]^{-},(\Delta=1.9 \mathrm{ppm})$.

ZGlyGlyGlyOH: A similar procedure to that described for ZGlyGlyOH was used. The starting material in this case was GlyGlyGly (435 mg, $2.3 \mathrm{mmol})$. White crystals were obtain $(0.72 \mathrm{~g}$, $2.23 \mathrm{mmol}, 97 \%){ }^{1} \mathrm{H}$ NMR (500 MHz, 30 ${ }^{\circ} \mathrm{C}, \mathrm{d}_{6}$-DMSO) $\delta 8.12$ $(\mathrm{m}, 2 \mathrm{H}), 7.45(\mathrm{t}, J=5.4 \mathrm{~Hz}, 1 \mathrm{H}), 7.33(\mathrm{~m}, 4 \mathrm{H}), 5.03(\mathrm{~s}, 2 \mathrm{H}), 3.75$ $(\mathrm{dd}, J=5.4 \mathrm{~Hz}, 3 \mathrm{H}), 3.66(\mathrm{~d}, J=6.0 \mathrm{~Hz}, 2 \mathrm{H}) .{ }^{13} \mathrm{C} \mathrm{NMR}(75 \mathrm{MHz}$, $30^{\circ} \mathrm{C}, \mathrm{d}_{6}$-DMSO) $\delta 171.5,169.8,169.6,156.9,137.4,128.8,128.2$, 128.2, 66.0, 44.0, 42.2, 41.0 . (ESI-TOF, negative mode) $\mathrm{m} / \mathrm{z}$ exp $[\mathrm{M}-\mathrm{H}]^{-}$calcd for $\mathrm{C}_{14} \mathrm{H}_{16} \mathrm{~N}_{3} \mathrm{O}_{6}{ }^{-} 322.1039$; found, $322.1034[\mathrm{M}$ $\mathrm{H}]^{-},(\Delta=1.6 \mathrm{ppm})$.

\subsection{Kinetic studies.}

All the experiments were carried out at $30^{\circ} \mathrm{C}$. [Substrate] $=15 \mathrm{mM}$, $[\mathrm{NaOH}]=0.1 \mathrm{M}$. The hydrolysis was followed by ${ }^{1} \mathrm{H}$ NMR $(500$ $\mathrm{MHz}$ ) monitoring the disappearance of the benzylic protons of the carbamate moiety. The data obtained by NMR at different times were adjusted to pseudo first order kinetics considering the concentration of hydroxide approximately constant in the studied time interval. Half-life times were calculated from the kinetic constant $\left(\mathrm{t}_{1 / 2}=\ln 2 / \mathrm{k}\right)$

\subsection{Racemization study}

PheNHPr was prepared by Z-removal with hydrogenolysis and by treatment with $\mathrm{NaOH}$ in methanol and the optical rotation compared.

PheNHPr obtained by basic treatment: Compound ZPheNHPr (10.2 mg, $0.03 \mathrm{mmol}$ ) was dissolved in $\mathrm{CD}_{3} \mathrm{OD} 0.1 \mathrm{M}$ of $\mathrm{NaOH}$ The reaction was monitorized by ${ }^{1} \mathrm{H}$ NMR. When the reaction was completed, the solvent was removed under reduced pressure. The crude was dissolved in $\mathrm{CH} 2 \mathrm{Cl} 2(10 \mathrm{~mL})$ and washed with water $(2 \times 10 \mathrm{~mL})$ and brine $(10 \mathrm{~mL})$. Organic layer was dried with $\mathrm{MgSO} 4$ anhidrous and the solvent was removed under reduced pressure $\left(6 \mathrm{mg}, 0.029 \mathrm{mmol}, 95 \%,[\alpha]_{\mathrm{D}}=0, \mathrm{c} 0.1, \mathrm{CHCl} 3\right)$.

PheNHPr obtained by hydrogenolysis. Palladium catalyst (10\% w/w, $\mathrm{Pd} / \mathrm{C}, 30 \mathrm{mg})$ was suspended in $\mathrm{MeOH}(10 \mathrm{~mL})$ and stirred under $\mathrm{H}_{2}$ at room temperature for $10 \mathrm{~min}$. Subsequently, a solution of compound ZPheNHPr, (300 mg, $0.88 \mathrm{mmol})$ in $\mathrm{MeOH}(20 \mathrm{~mL})$ was added via syringe, followed by stirring under $\mathrm{H}_{2}$ at room temperature for $4 \mathrm{~h}$. The reaction mixture was then filtered through Celite, and the solvent was removed under reduced pressure (172 $\left.\mathrm{mg}, 0.83 \mathrm{mmol}, 95 \%,[\alpha]_{\mathrm{D}}=-86, c 0.3, \mathrm{CHCl}_{3}\right)$.

${ }^{1} \mathrm{H}$ NMR (500 MHz, $30{ }^{\circ} \mathrm{C}, \mathrm{d}_{6}$-DMSO) $\delta 7.71(\mathrm{t}, J=5.0 \mathrm{~Hz}, 2 \mathrm{H})$ $7.27-7.21(\mathrm{~m}, 2 \mathrm{H}), 7.20-7.14(\mathrm{~m}, 3 \mathrm{H}), 3.33(\mathrm{dd}, J=7.9,5.4 \mathrm{~Hz}$ $1 \mathrm{H}), 3.06-2.91(\mathrm{~m}, 2 \mathrm{H}), 2.91-2.80(\mathrm{~m}, 2 \mathrm{H}), 2.65-2.52(\mathrm{~m}, 2 \mathrm{H})$ $1.42-1.24(\mathrm{~m}, 2 \mathrm{H}), 0.76(\mathrm{t}, J=7.4 \mathrm{~Hz}, 3 \mathrm{H}){ }^{13} \mathrm{C}$ NMR $(75 \mathrm{MHz}$ $\left.\mathrm{CDCl}_{3}\right) \delta 173.3,137.2,128.5,127.8,125.9,55.7,40.3,40.0,22.0$, 10.6. (ESI-TOF, positive mode) $\mathrm{m} / \mathrm{z}$ exp $[\mathrm{M}+\mathrm{H}]^{+}$calcd for $\mathrm{C}_{12} \mathrm{H}_{19} \mathrm{~N}_{2} \mathrm{O}^{+}$207.1497; found, $207.1497[\mathrm{M}+\mathrm{H}]^{+},(\Delta=0.0 \mathrm{ppm})$. ((Experimental Details))

Supporting Information (see footnote on the first page of this article): ${ }^{1} \mathrm{H}$ and ${ }^{13} \mathrm{C}$ NMR spectra, synthetic schemes and details of kinetic experiments

Acknowledgments. We thank Spanish Ministry of Science and Innovation (grant CTQ2012-37735) and Universitat Jaume I (grant P1.1B2012-25) for financial support. M.T.-S. thanks Spanish Ministry of Education for a FPU fellowship

[1] D. V. Titov, M. L. Gening, A. G.; Gerbst, A. O. Chizhov, Y. E Tsvetkov, N. E. Nifantiev, Carbohyd. Res. 2013, 381, 161. 
[2] B. M. Fraga, V. González-Vallejo, C. Bressa, R. Guillermo, S. Suárez, Tetrahedron 2013, 69, 3002.

[3] T. Ohwadaa, N. Tania, Y. Sakamakia, Y. Kabasawaa, Y. Otania, M.Kawahatab, K. Yamaguchi, Proc. Nat. Acad. Sci. doi: 10.1073/pnas.1300381110

[4] G. Fibriansah, F. I. Gliubich, A.-M. W. H. Thunnissen, Biochem., 2012, 51, 9164 .

[5] M. Maiti, S. Michielssens, N. Dyubankova, M. Maiti, . E. Lescrinier, A. Ceulemans, P. Herdewijn, Chem. Eur. J. 2012, 18, 857-868.

[6] F. Aricò, M. Chiurato, J. Peltier, P. Tundo, Eur. J. Org. Chem. 2012, 3223 .

[7] P. J. Kocienski, Protecting Groups, Georg Thieme Verlag, Stuttgart, New York, 2005

[8] A. Isidro-Llobet, M. Álvarez, F. Albericio, Chem. Rev. 2009, 109, 2455 .

[9] P. G. M. Wuts, T. W.; Greene, Protective Groups in Organic Synthesis, Wiley-Interscience: New York, 2006.

[10] L. E. Overman, M. J. Sharp, Tetrahedron Lett. 1988, 29, 901.

[11] S. R. Angle, D. O. Arnaiz, Tetrahedron Lett. 1989, 30, 515.

[12] V. Castelletto, I. W Hamley, C. Cenker, U. Olsson, J. Adamcik, R. Mezzenga, J. F. Miravet, B. Escuder, F. Rodriguez-Llansola, J. Phys. Chem. B 2011, 115, 2107

[13] V. J. Nebot, J. Armengol, J. Smets, S. F. Prieto, B. Escuder, J. F Miravet, Chem. Eur. J. 2012, 18, 4063
[14] Although the labile nature of $\mathrm{Z}$ groups in mild basic media could be of evident interest in peptide synthesis, unfortunately epimerization/racemization was detected in the Z-depleted products (see experimental section).

[15] M. L. Bender, R. B. Homer, J. Org. Chem. 1965, 30, 3975.

[16] I. Christenson, Acta Chem.Scand. 1964, 18, 904.

[17] A. Williams, J. Chem. Soc. Perkin Trans. 2 1972, 808.

[18] D. Nalbantova, D. Cheshmedzhieva, B. Hadjieva, S. Ilieva, B Galabov, J. Phys. Org. Chem. 2011, 24, 1166.

[19] W. P Jencks, Catalysis in Chemistry and Enzymology, Dover Publications, New York, 1986.

[20] A.; Warshel, P. K.; Sharma, M. Kato, Y. Xiang, H. Liu, M. H. M. Olsson, Chem. Rev. 2006, 106, 3210.

[21] Escuder, B.; Martí, S.; Miravet, J.F. Langmuir, 2005, 21, 6776.

[22] H. Herzner, H. Kunz Carbohyd. Res., 2007, 342, 541.

[23] B. Yde, I. Thomsen, M. Thorsen, K. Clausen, S.O. Lawesson, Tetrahedron 1983, 39, 4121.

[24] F. S.; Gibson, M. S. Park, H. Rapoport J.Org. Chem., 1994, 59, 7505.

Received: ((will be filled in by the editorial staff)) Published online: ((will be filled in by the editorial staff) 
Entry for the Table of Contents ((Please choose one layout.))

\section{Layout 1:}

((Key Topic) $)$

$\mathrm{Z}$ group from $\mathrm{N}$-protected peptides is found to be extremely labile in diluted aqueous sodium hydroxide. The key for this unexpected behaviour is the anchimeric assistance by the vicinal amide NH. Amazingly, this assistance is blocked in dipeptides due to an intramolecular H-bonding with the terminal carboxylate.

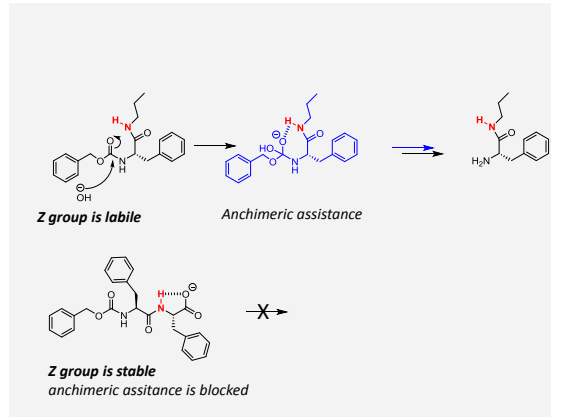

Marta Tena-Solsona, César A. Angulo-Pachón, Beatriu Escuder* and Juan F. Miravet*.

Mechanistic Insight into the Lability of Benzyloxycarbonyl (Z) Group in NProtected Peptides under Mild Basic Conditions

Keywords: Anchimeric assistance / neighbouring group participation /

\section{Layout 2:}

((Please adjust TOC Graphic to the size of this area; max. width $11.5 \mathrm{~cm}$; max. height $2.5 \mathrm{~cm})$ )

((Text for Table of Contents))
((Text for Table of Contents, Continued - max. 350 characters; not the same text as the Abstract))
((Author(s), with * for Corresponding Author(s))) ......... Page No. - Page No.

((Title))

Keywords: ((Keyword 1 / Keyword 2 / Keyword 3 / Keyword 4 / Keyword 5)) 


\section{Supporting Information}

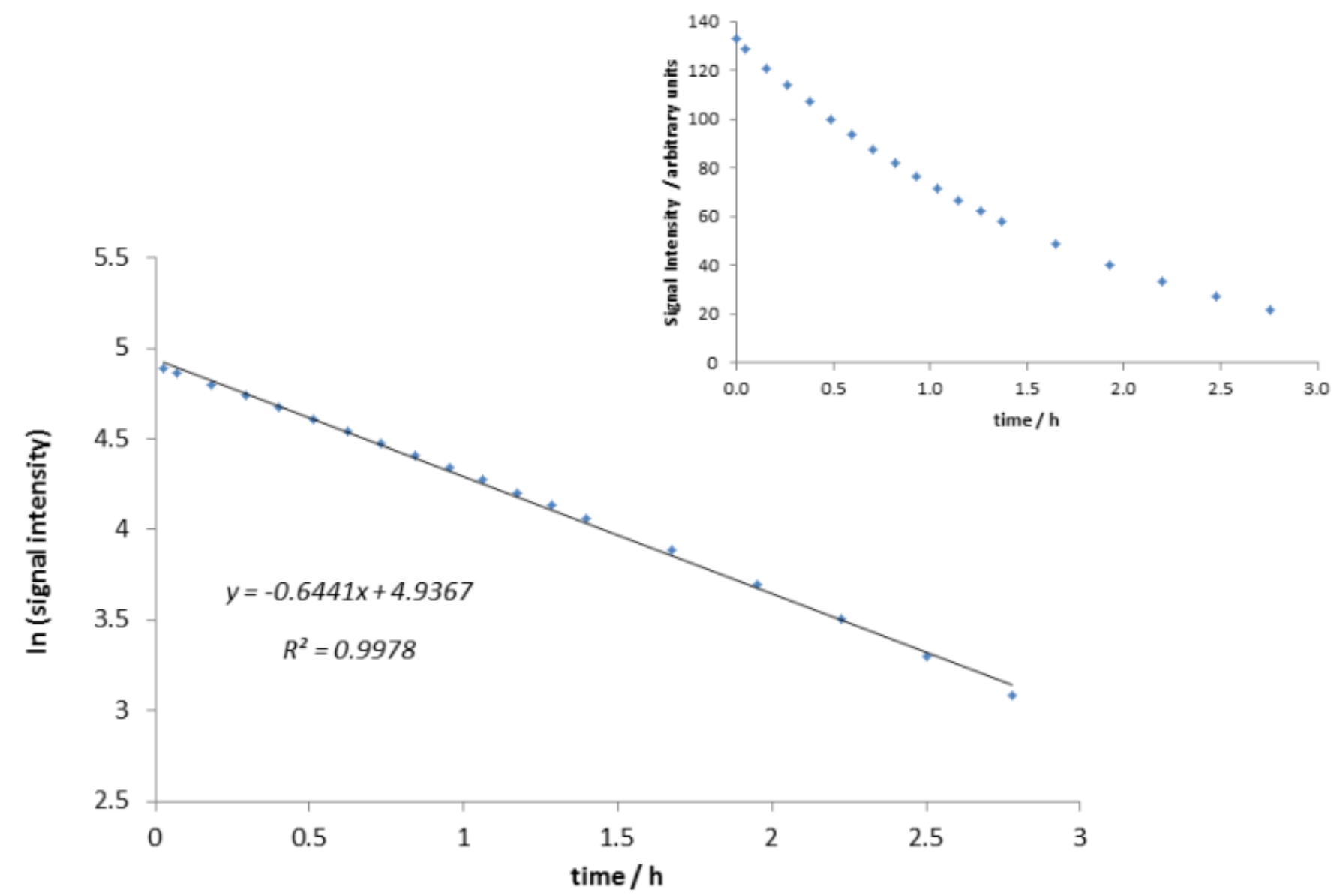

Figure S1. Graphical representation of the kinetic data obtained from NMR for the hydrolysis of ZDFFDNHPr in $\mathrm{D}_{2} \mathrm{O}$. 
Compounds, ZPheNHPr, ZValNHPr, ZSarNHPr and ZPheN $\left(\mathbf{C H}_{3}\right) \mathbf{P r}$ were prepared following the steps shown in scheme S1.

\section{SCHEME S1}

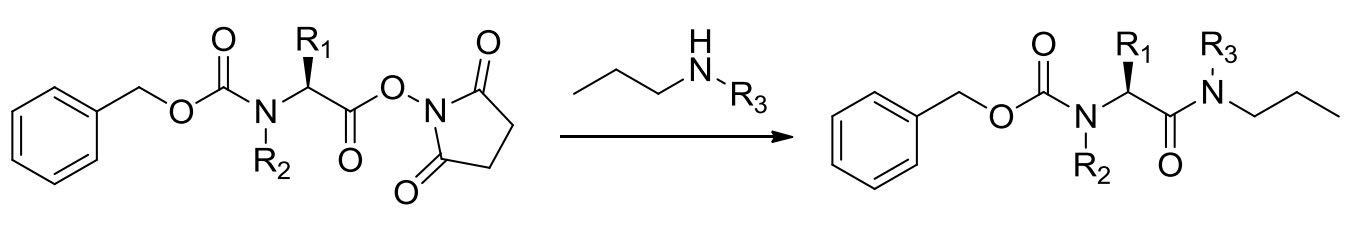

ZPheNHPr: $\mathrm{R}_{1}=-\mathrm{CH}_{2} \mathrm{Ph}, \mathrm{R}_{2}=-\mathrm{H}, \mathrm{R}_{3}=-\mathrm{H}$

ZValNHPr: $\mathrm{R}_{1}=-\mathrm{CH}\left(\mathrm{CH}_{3}\right)_{2}, \mathrm{R}_{2}=-\mathrm{H}, \mathrm{R}_{3}=-\mathrm{H}$

ZSarNHPr: $\mathrm{R}_{1}=-\mathrm{H}, \mathrm{R}_{2}=-\mathrm{CH}_{3}, \mathrm{R}_{3}=-\mathrm{H}$

ZPheN $\left(\mathrm{CH}_{3}\right) \operatorname{Pr}: \mathrm{R}_{1}=-\mathrm{CH}_{2} \mathrm{Ph}, \mathrm{R}_{2}=-\mathrm{H}, \mathrm{R}_{3}=-\mathrm{CH}_{3}$

Compounds, ZAspNHPr, ZAspN $\left(\mathbf{C H}_{3}\right)$ Pr were prepared following the steps shown in scheme S2.

\section{SCHEME S2}
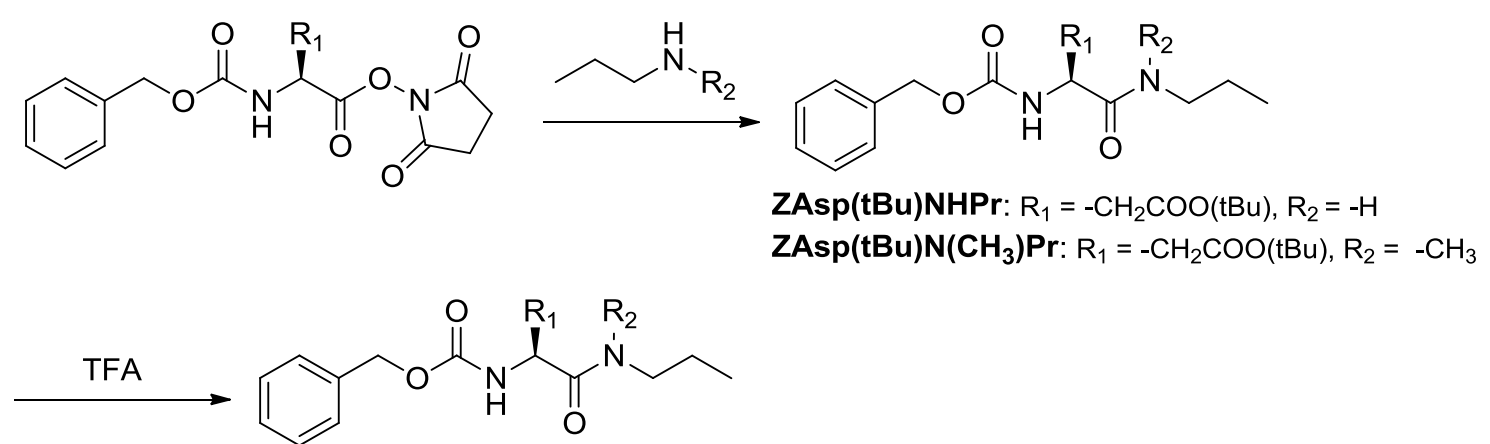

ZAspNHPr: $\mathrm{R}_{1}=-\mathrm{CH}_{2} \mathrm{COOH}, \mathrm{R}_{2}=-\mathrm{H}$

$\mathrm{ZAspN}\left(\mathrm{CH}_{3}\right) \mathrm{Pr}: \mathrm{R}_{1}=-\mathrm{CH}_{2} \mathrm{COOH}, \mathrm{R}_{2}=-\mathrm{CH}_{3}$ 
Compounds, ZPhePheOH and ZAsp(tBu)PheOH were prepared following the steps shown in scheme S3.

\section{SCHEME S3}

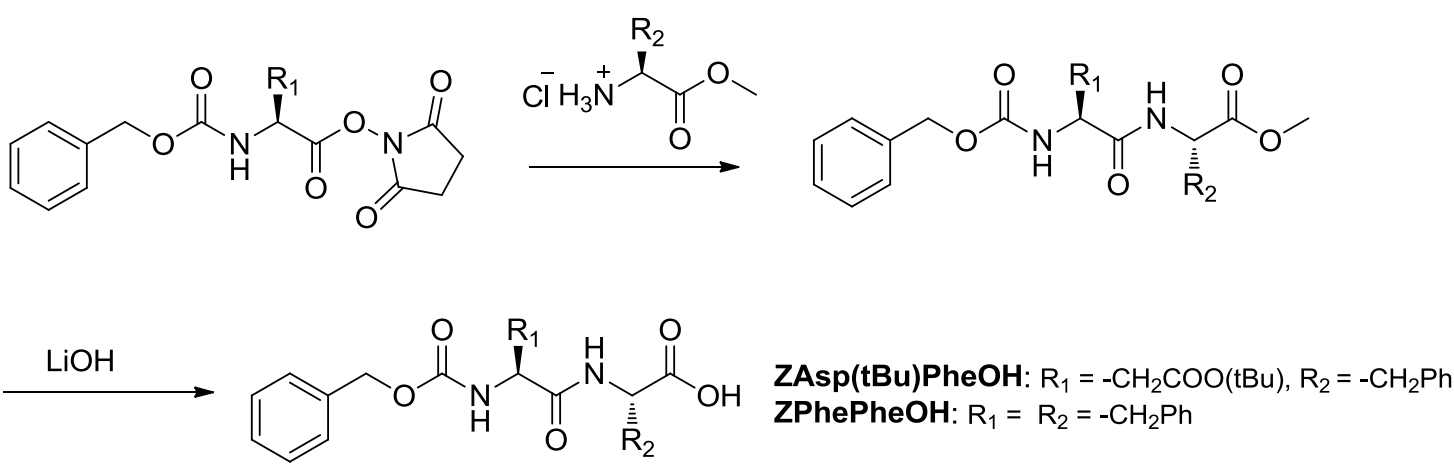


Compounds, ZPheAspAspPheNHPr and ZAspPheAspPheNHPr and ZAspPhePheAspNHPr were prepared following the steps shown in scheme S4.

SCHEME S4

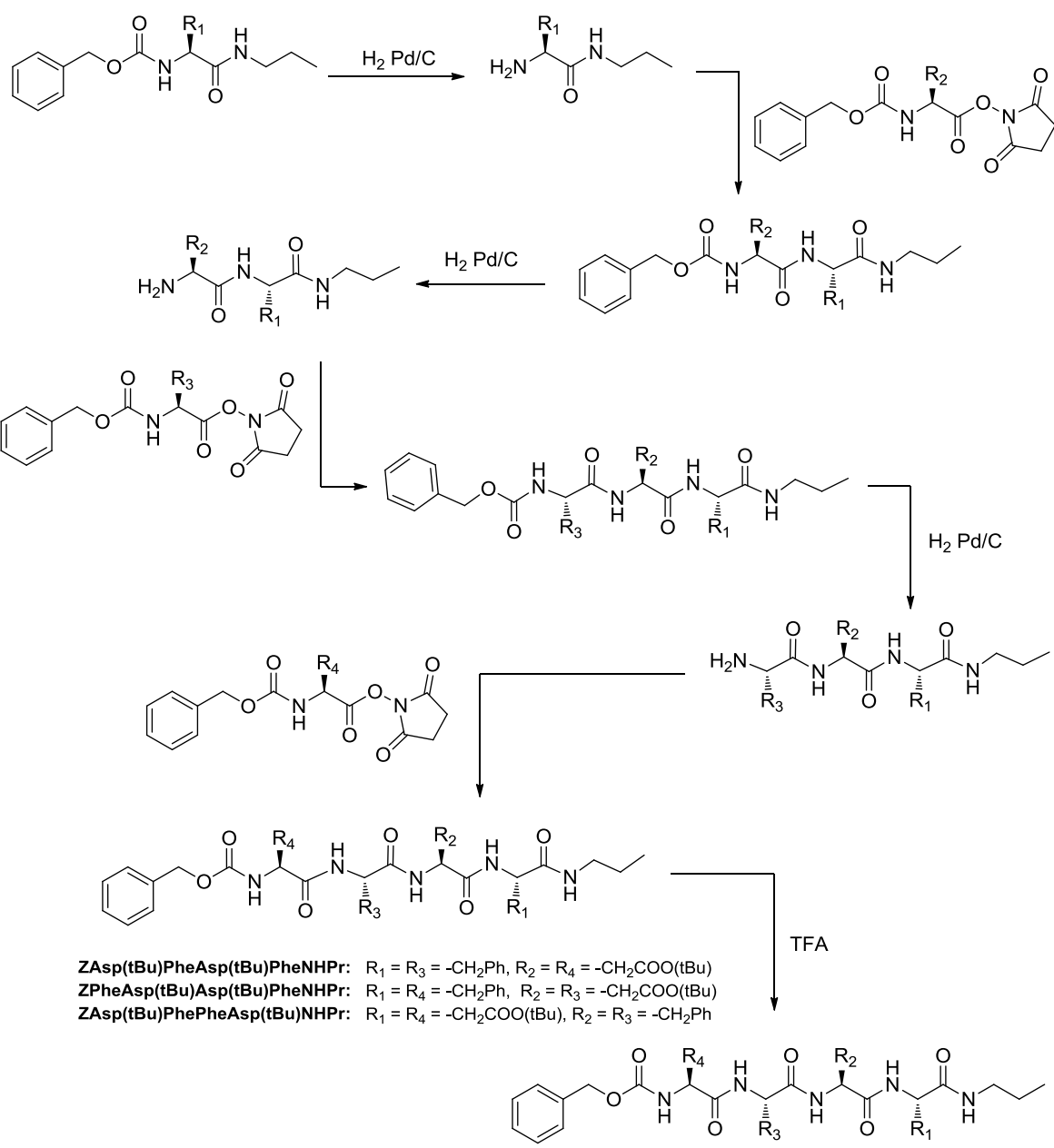

ZAspPheAspPheNHPr: $\mathrm{R}_{1}=\mathrm{R}_{3}=-\mathrm{CH}_{2} \mathrm{Ph}, \mathrm{R}_{2}=\mathrm{R}_{4}=-\mathrm{CH}_{2} \mathrm{COOH}$ ZPheAspAspPheNHPr: $R_{1}=R_{4}=-\mathrm{CH}_{2} \mathrm{Ph}, \mathrm{R}_{2}=\mathrm{R}_{3}=-\mathrm{CH}_{2} \mathrm{COOH}$
ZAspPhePheAspNHPr: $R_{1}=R_{4}=-\mathrm{CH}_{2} \mathrm{COOH}, \mathrm{R}_{2}=\mathrm{R}_{3}=-\mathrm{CH}_{2} \mathrm{Ph}$ 


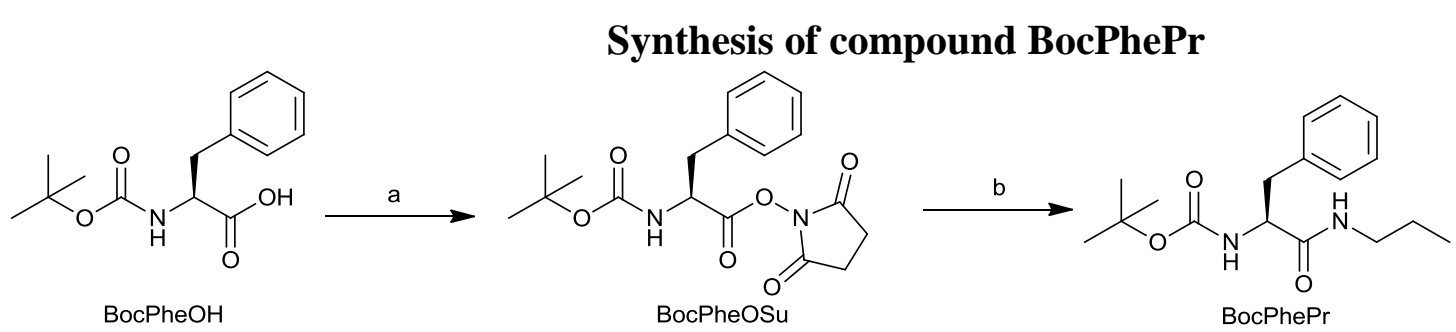

Scheme S5. Reagents and conditions: a) DCC, $N$-Hydroxisuccinimide, DME, $0{ }^{\circ} \mathrm{C}, 1 \mathrm{~h}, 98 \%$; b) $n$-Propylamine, THF, room temperature, $16 \mathrm{~h}, 96 \%$.

Synthesis of compound $(S)$-2,5-dioxopyrrolidin-1-yl 2-((tert-butoxycarbonyl)amino)-3phenylpropanoate (BocPheOSu): A solution of commercial available tert-butoxycarbonyl- $L$ phenylalanine BocPheOH (2.0 g, $7.53 \mathrm{mmol})$ and $N$-hydroxysuccinimide $(867 \mathrm{mg}, 7.53 \mathrm{mmol}, 1.0$ eq.) in DME $(30 \mathrm{~mL})$ was added dropwise under $\mathrm{N}_{2}$ at $0{ }^{\circ} \mathrm{C}$ with a dropping funnel to a solution of $N, N^{\prime}-$ Dicyclohexylcarbodiimide (1.7 g, $8.28 \mathrm{mmol}, 1.01 \mathrm{eq}$.) in dry DME $(15 \mathrm{~mL})$. The mixture was further stirred for $1 \mathrm{~h}$ at $0{ }^{\circ} \mathrm{C}$. The solution was then allowed to stand into refrigerator for $16 \mathrm{~h}$, which caused precipitation of $N, N^{\prime}$-Dicyclohexylurea. After this time, the mixture was filtered under vacuum, and the filtrate was removed under reduced pressure and the crude residue was purified by crystallization in isopropanol to yield BocPheOSu (2.7 g, $7.34 \mathrm{mmol}, 98 \%)$ as a white solid. The NMR spectra were consistent with those described in the literature (N. I., Howard; T. D. H., Bugg. Bioorg. Med. Chem. 2003, 11, 3083)

${ }^{1} \mathrm{H}$ NMR $\left(300 \mathrm{MHz}, 30^{\circ} \mathrm{C}, \mathrm{CDCl}_{3}\right): \delta 7.20-7.38(\mathrm{~m}, 5 \mathrm{H}), 4.96(\mathrm{br} \mathrm{s}, 2 \mathrm{H}), 3.21(\mathrm{dd}, 2 \mathrm{H}, J=4.1,11.8$ $\mathrm{Hz}), 2.82(\mathrm{~s}, 4 \mathrm{H}), 1.40(\mathrm{~s}, 9 \mathrm{H})$.

Synthesis of compound $(S)$-tert-butyl (1-oxo-3-phenyl-1-(propylamino)propan-2-yl)carbamate (BocPhePr): A solution of compound BocPheOSu $(2.0 \mathrm{~g}, 5.52 \mathrm{mmol})$ in THF (40 mL) was added dropwise under $\mathrm{N}_{2}$ at $25^{\circ} \mathrm{C}$ with a dropping funnel to a solution of commercial available $n$-Propylamine ( $499 \mu \mathrm{L}, 6.07 \mathrm{mmol}, 1.1 \mathrm{eq}$.) in THF $(10 \mathrm{~mL})$. The mixture was further stirred for $5 \mathrm{~h}$ at $50^{\circ} \mathrm{C}$. After this time, the solvent was removed under reduced pressure and the residue was poured into dissolution aq. $\mathrm{HCl} 0.1$ and worked up (extraction with $\mathrm{CH}_{3} \mathrm{Cl}$ ), removal of all volatiles under reduced pressure to yield BocPhePr (1.62 g, $5.30 \mathrm{mmol}, 96 \%)$ as a white solid. The compound was used in crude form for the next reaction. The NMR spectra were consistent with those described in the literature. (D., Tessaro; L., Cerioli; S., Servi; F., Viani; P., D’Arrigo. Adv. Synth. Catal. 2011, 353, 233.)

${ }^{1} \mathrm{H} \mathrm{NMR}\left(500 \mathrm{MHz}, 30^{\circ} \mathrm{C}, \mathrm{CDCl}_{3}\right): \delta 7.19-7.32(\mathrm{~m}, 5 \mathrm{H}), 5.69(\mathrm{br} \mathrm{s}, 1 \mathrm{H}), 5.08(\mathrm{br} \mathrm{s}, 1 \mathrm{H}), 4.26(\mathrm{~d}, 1 \mathrm{H}, J$ $=6.8 \mathrm{~Hz}), 3.18-2.97(\mathrm{~m}, 4 \mathrm{H}), 1.44-1.33(\mathrm{~m}, 11 \mathrm{H}), 0.80(\mathrm{t}, 9 \mathrm{H}, J=7.3 \mathrm{~Hz})$. 


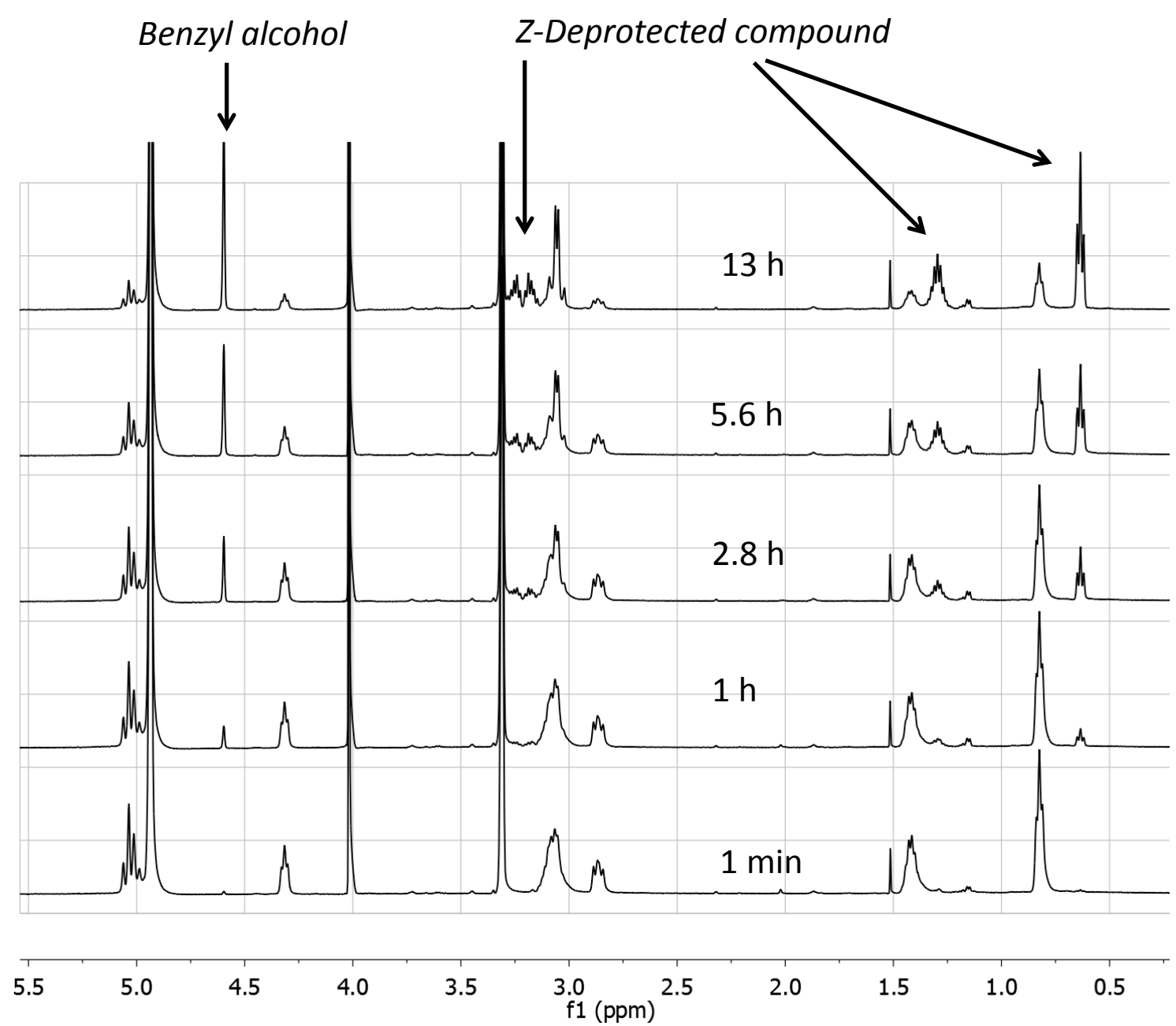

Figure S2. ${ }^{1} \mathrm{H}$ NMR spectra recorded for the basic hydrolysis of ZPheNHPr $(15 \mathrm{mM})$ in $\mathrm{CD}_{3} \mathrm{OD}$ $([\mathrm{NaOH}]=0.1 \mathrm{M})$. 


\section{${ }^{1} \mathrm{H}$ and ${ }^{13} \mathrm{C}$ NMR spectra for characterization purposes}




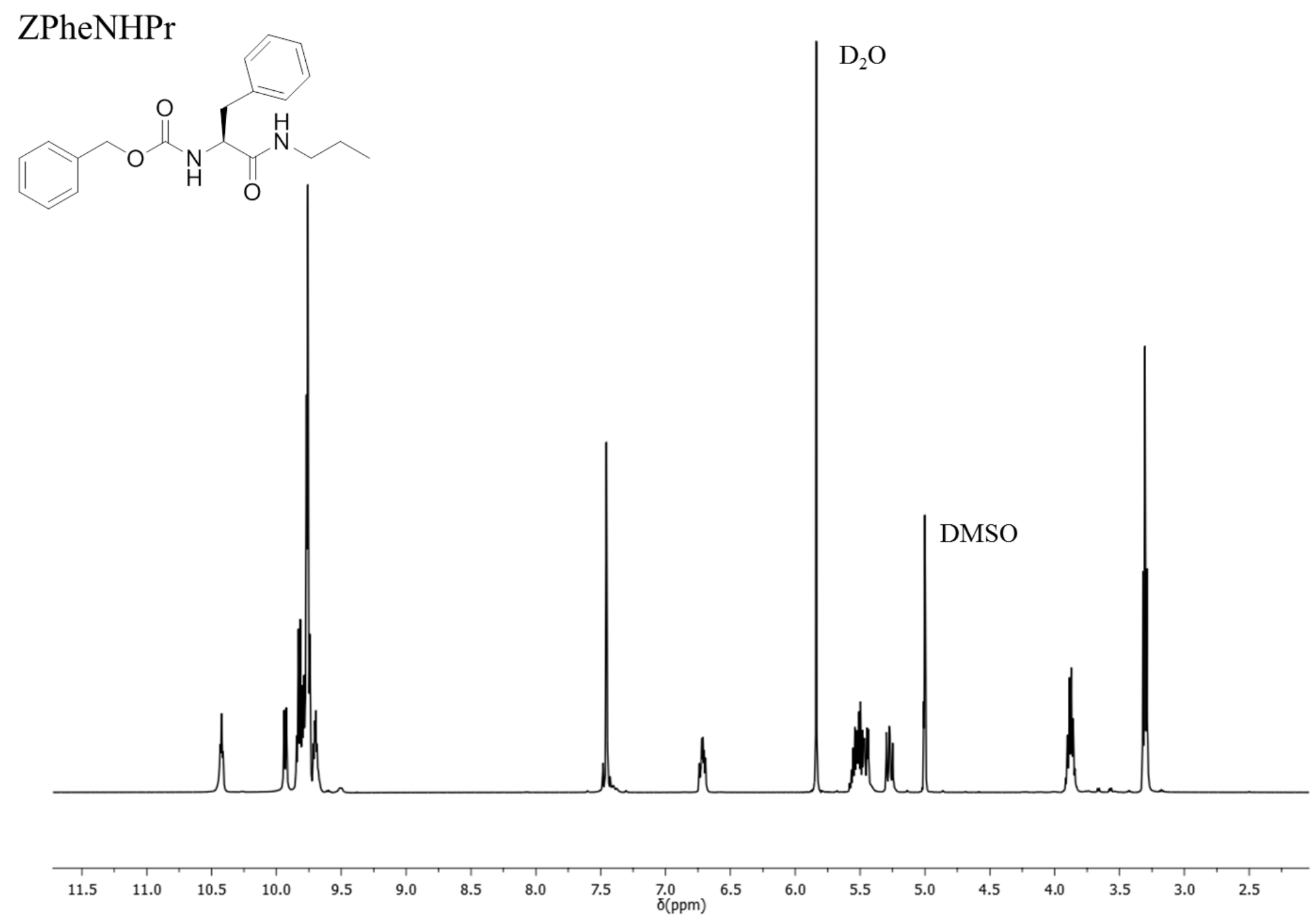



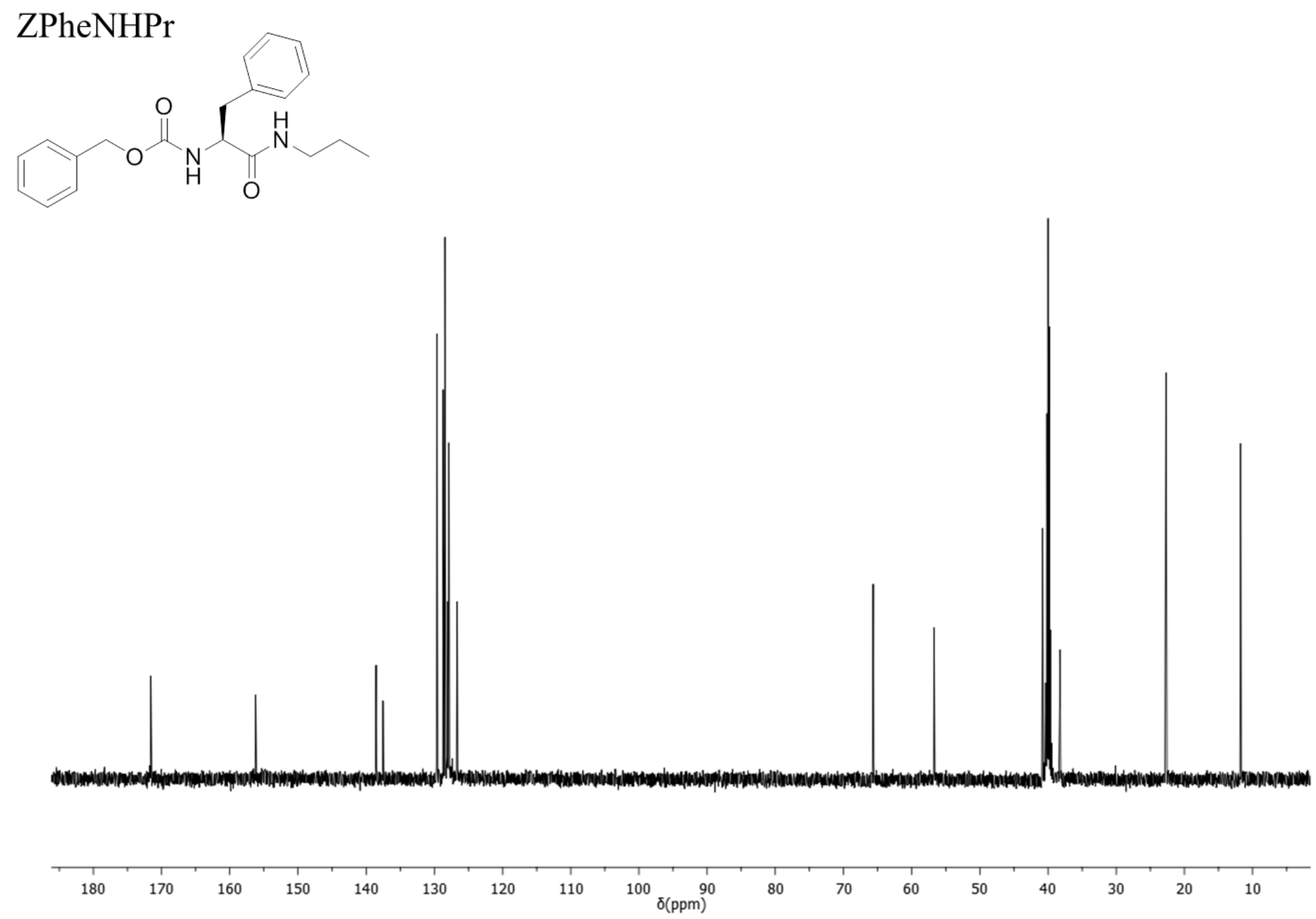


\section{ZValNHPr}

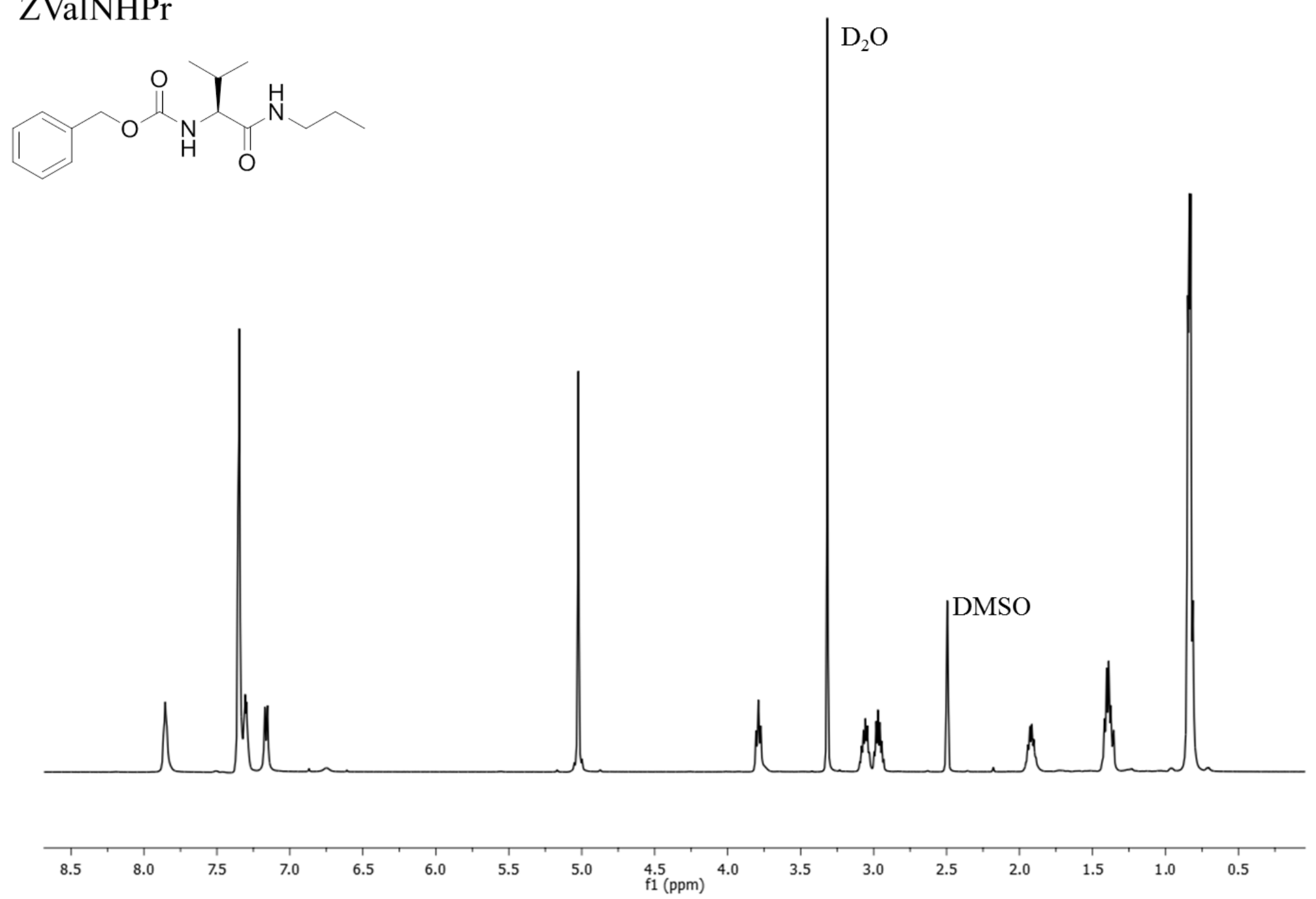




\section{ZValNHPr}

(1)

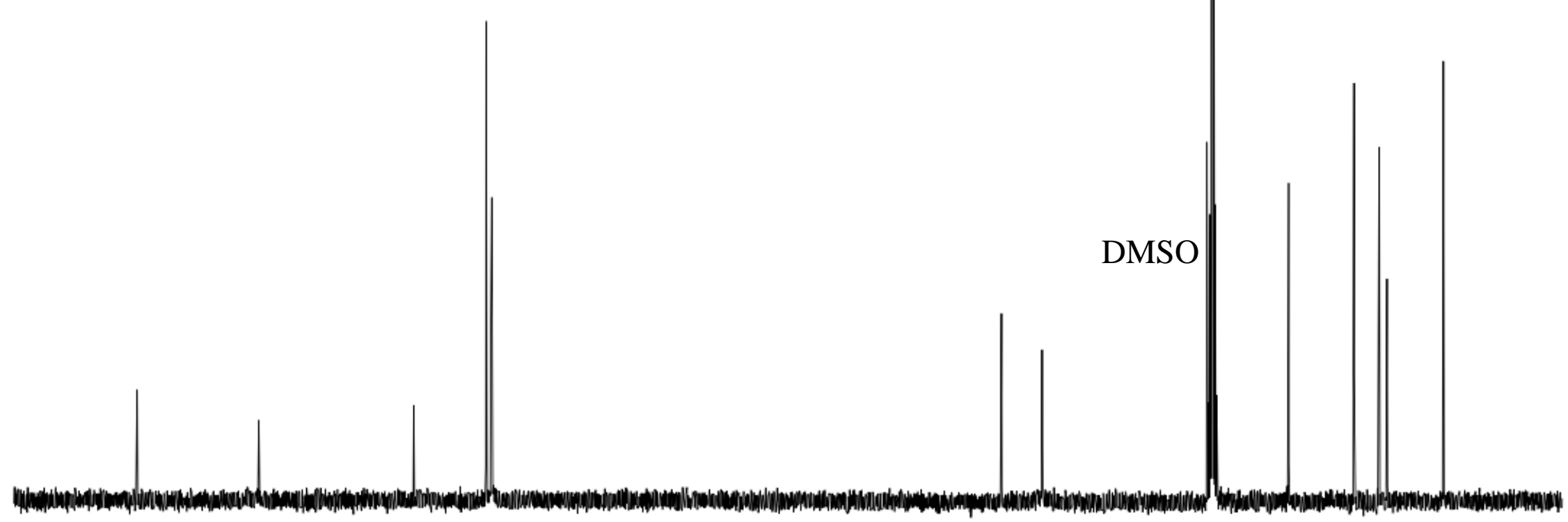

180 


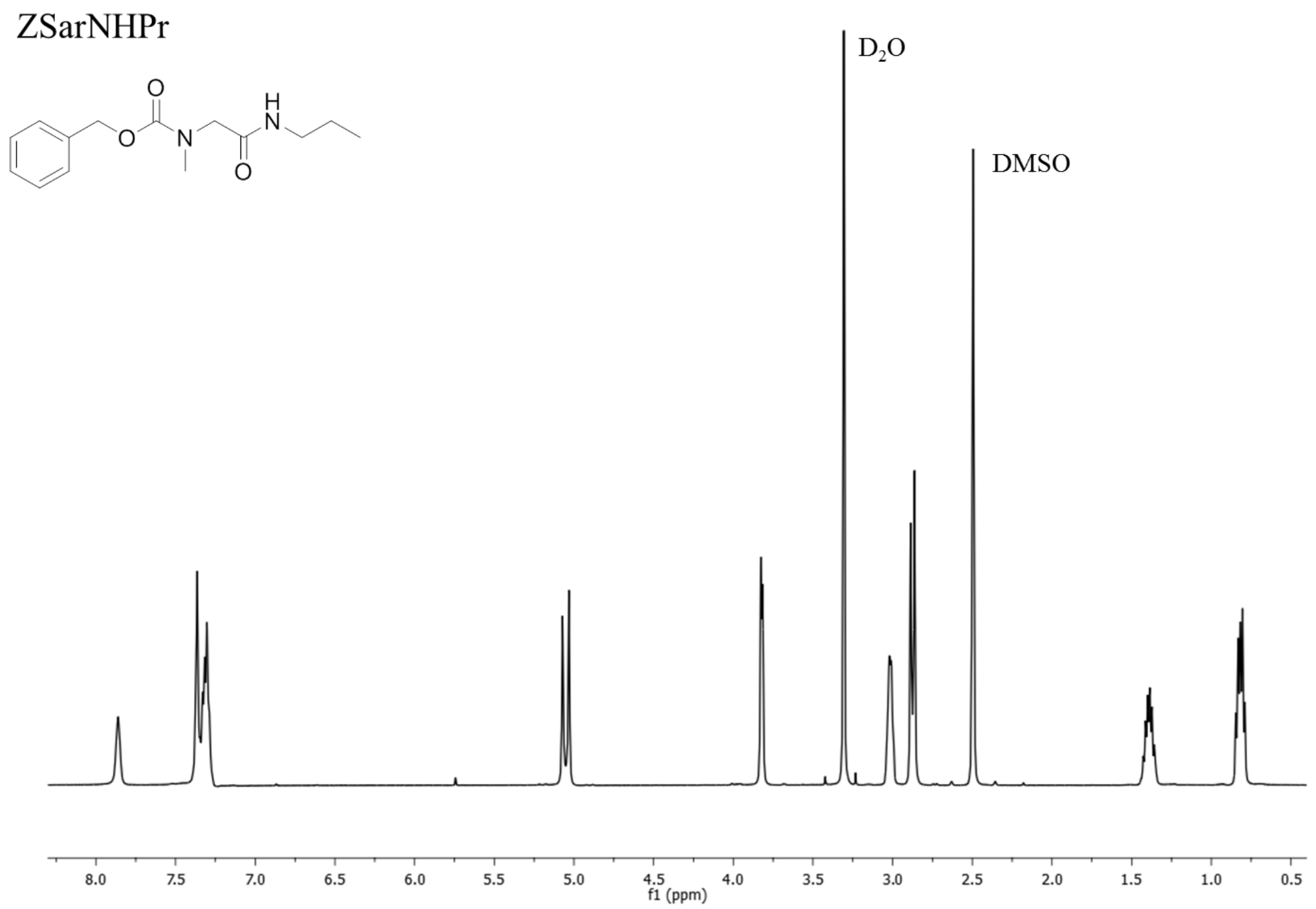




\section{ZSarNHPr}

$\mathrm{N}_{\mathrm{O}}^{\mathrm{N}} \mathrm{N}_{\mathrm{N}}^{\mathrm{O}}$
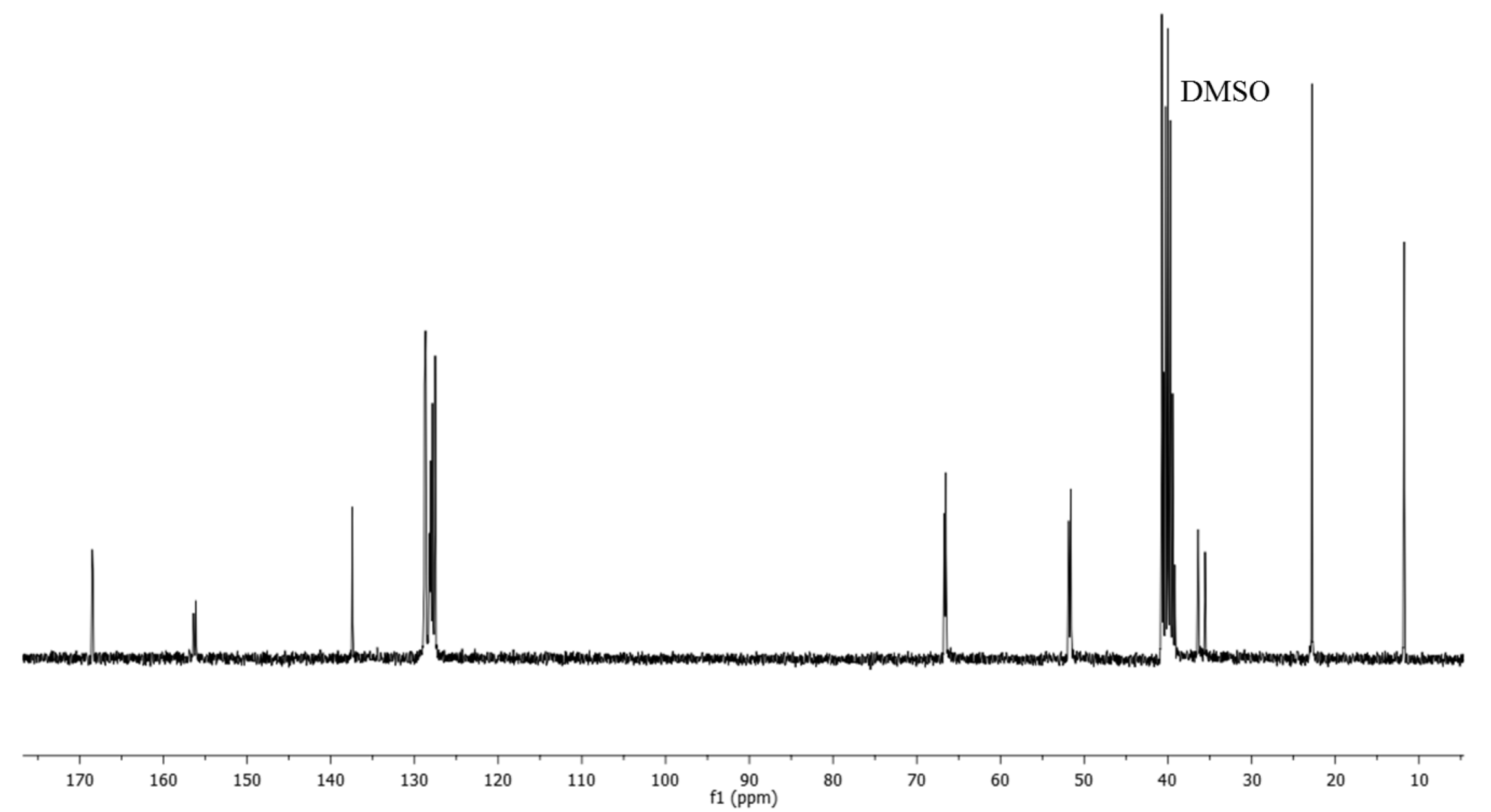


\section{$\mathrm{ZPheN}\left(\mathrm{CH}_{3}\right) \mathrm{Pr}$}

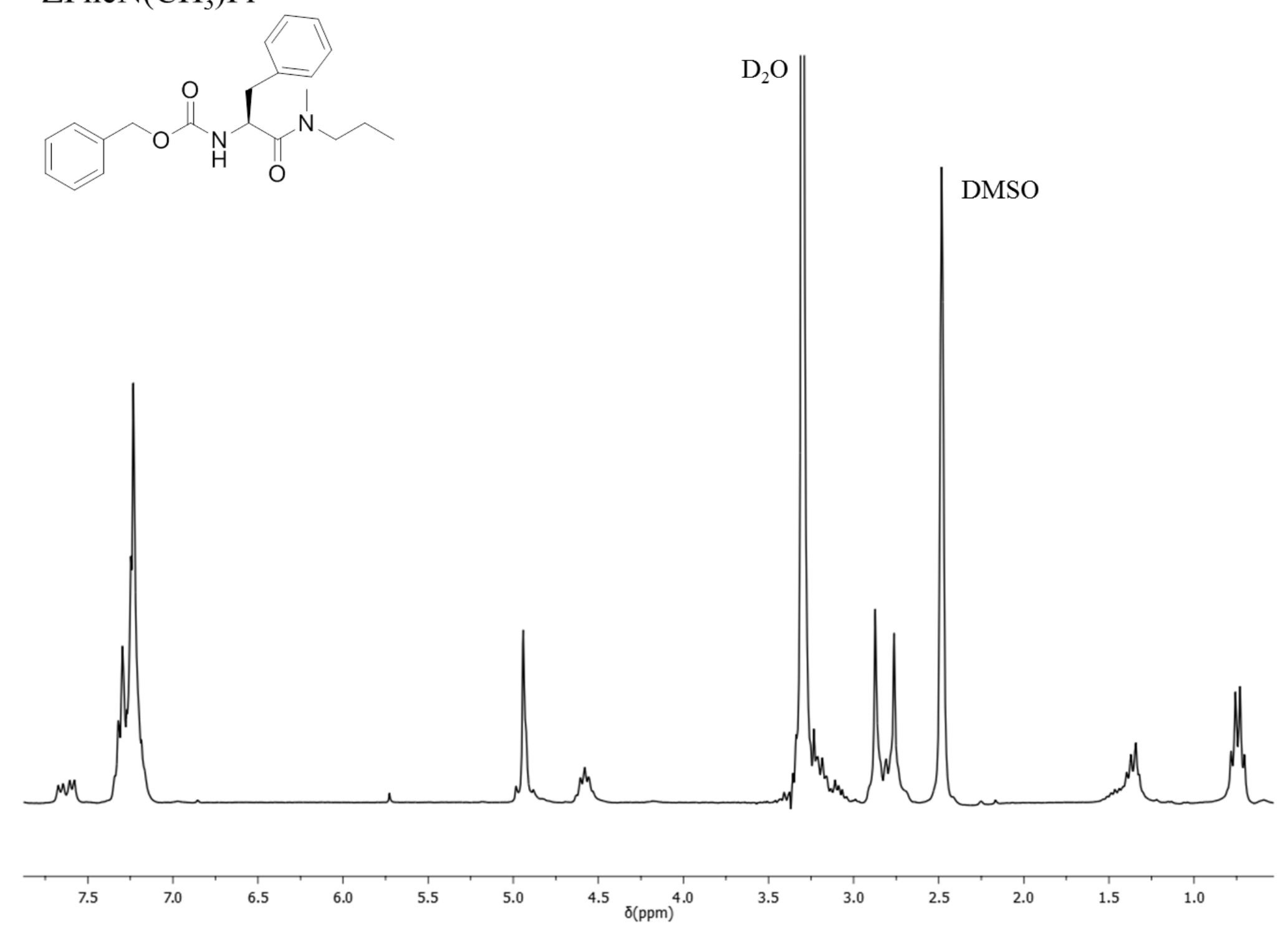




\section{ZPheN $\left(\mathrm{CH}_{3}\right) \mathrm{Pr}$}

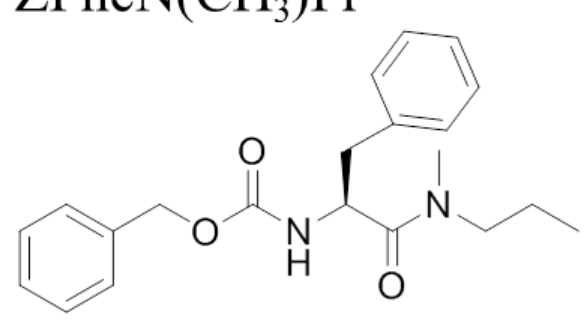

DMSO
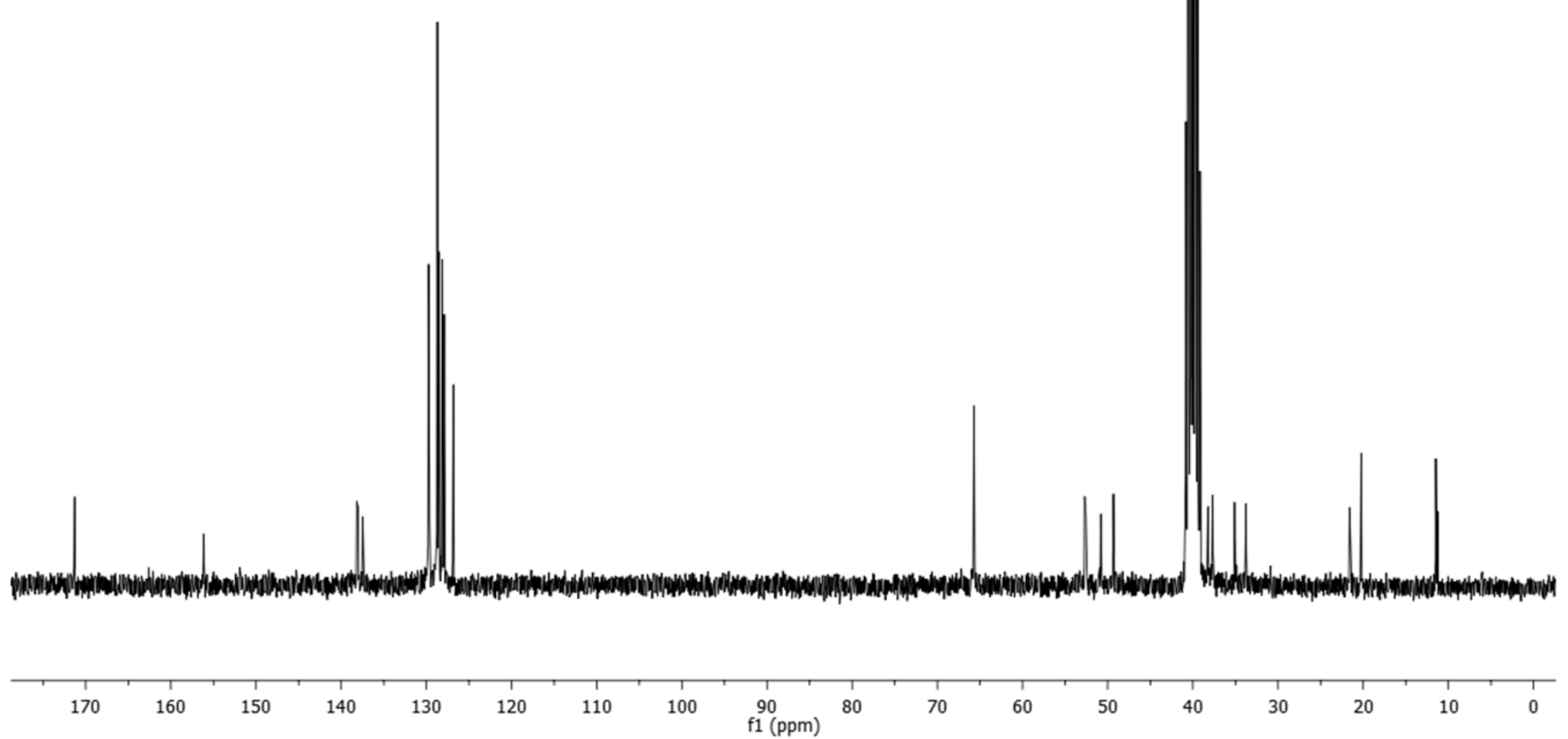


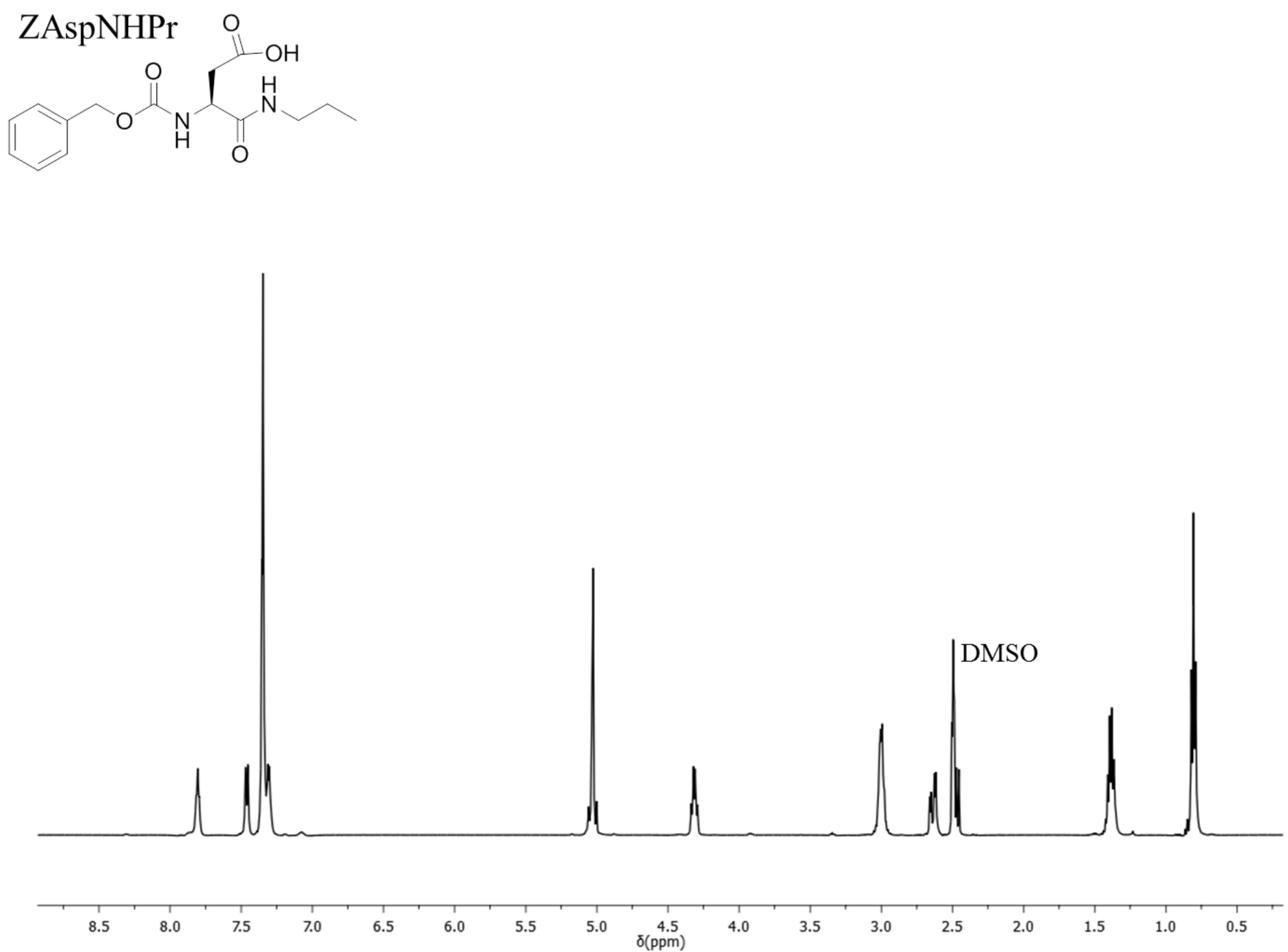



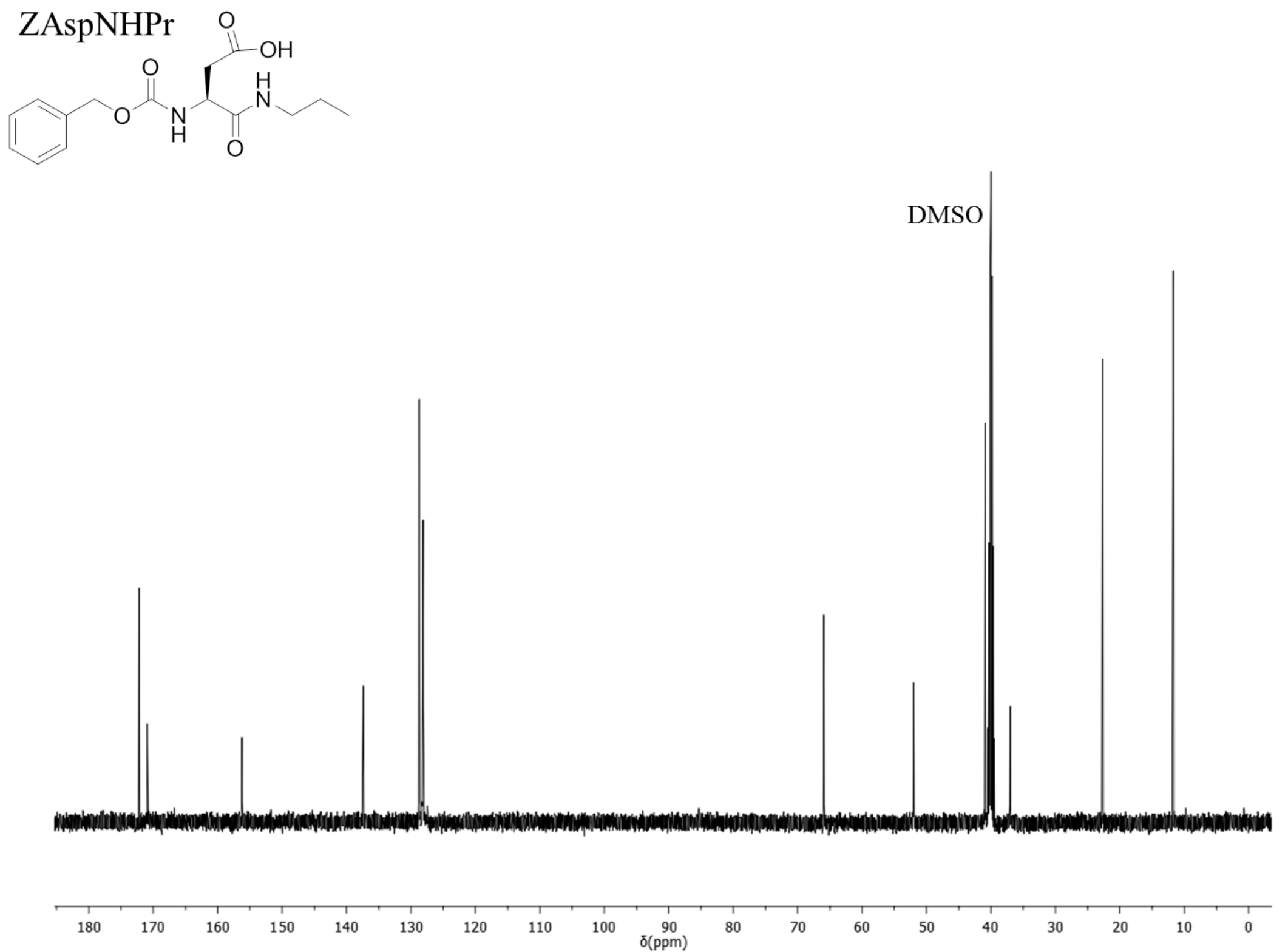


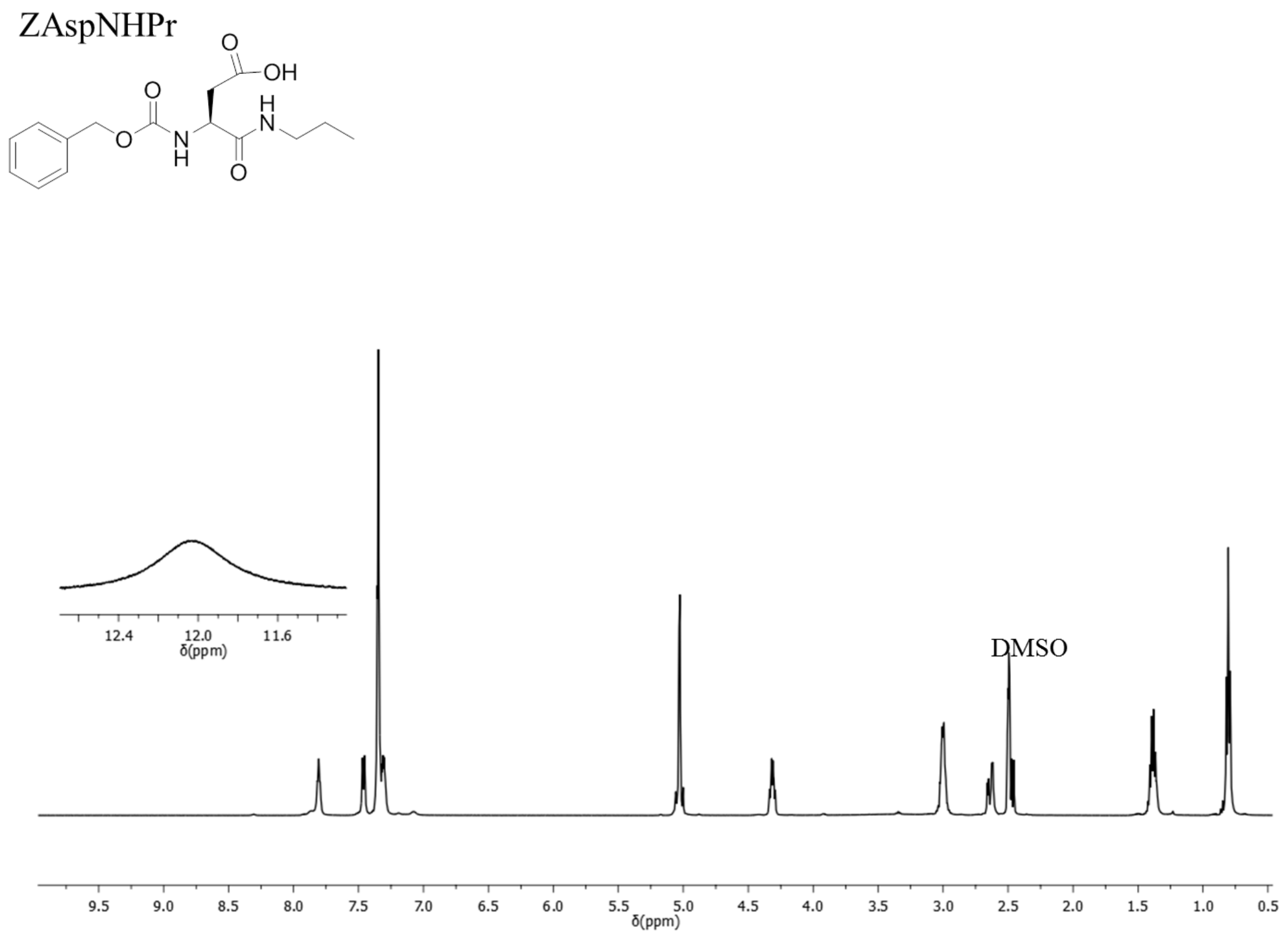




\section{ZAspN $\left(\mathrm{CH}_{3}\right) \mathrm{Pr}$}

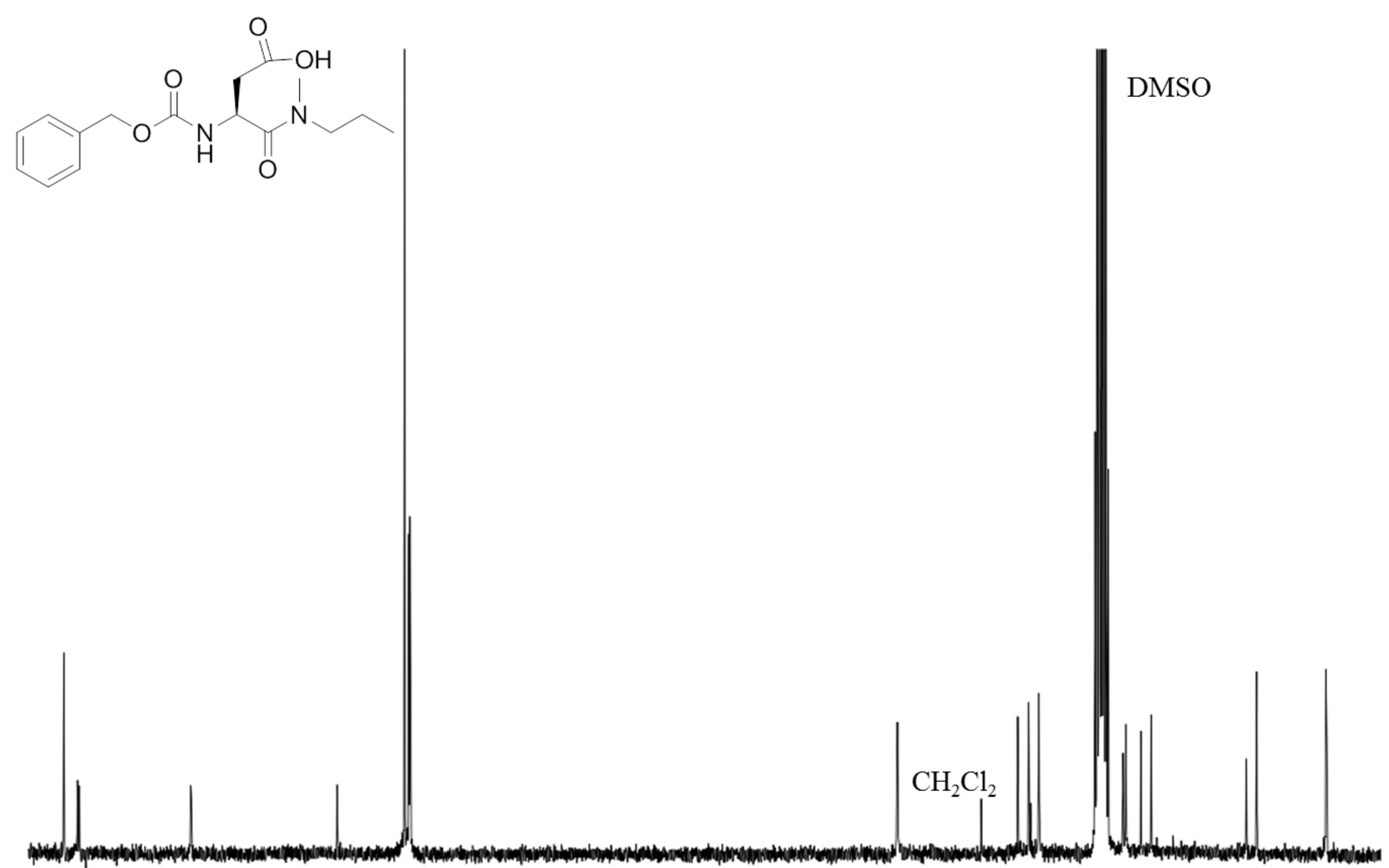

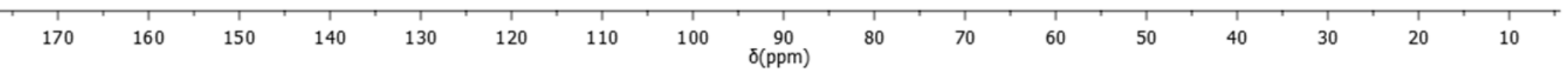




\section{ZPhePheOH}

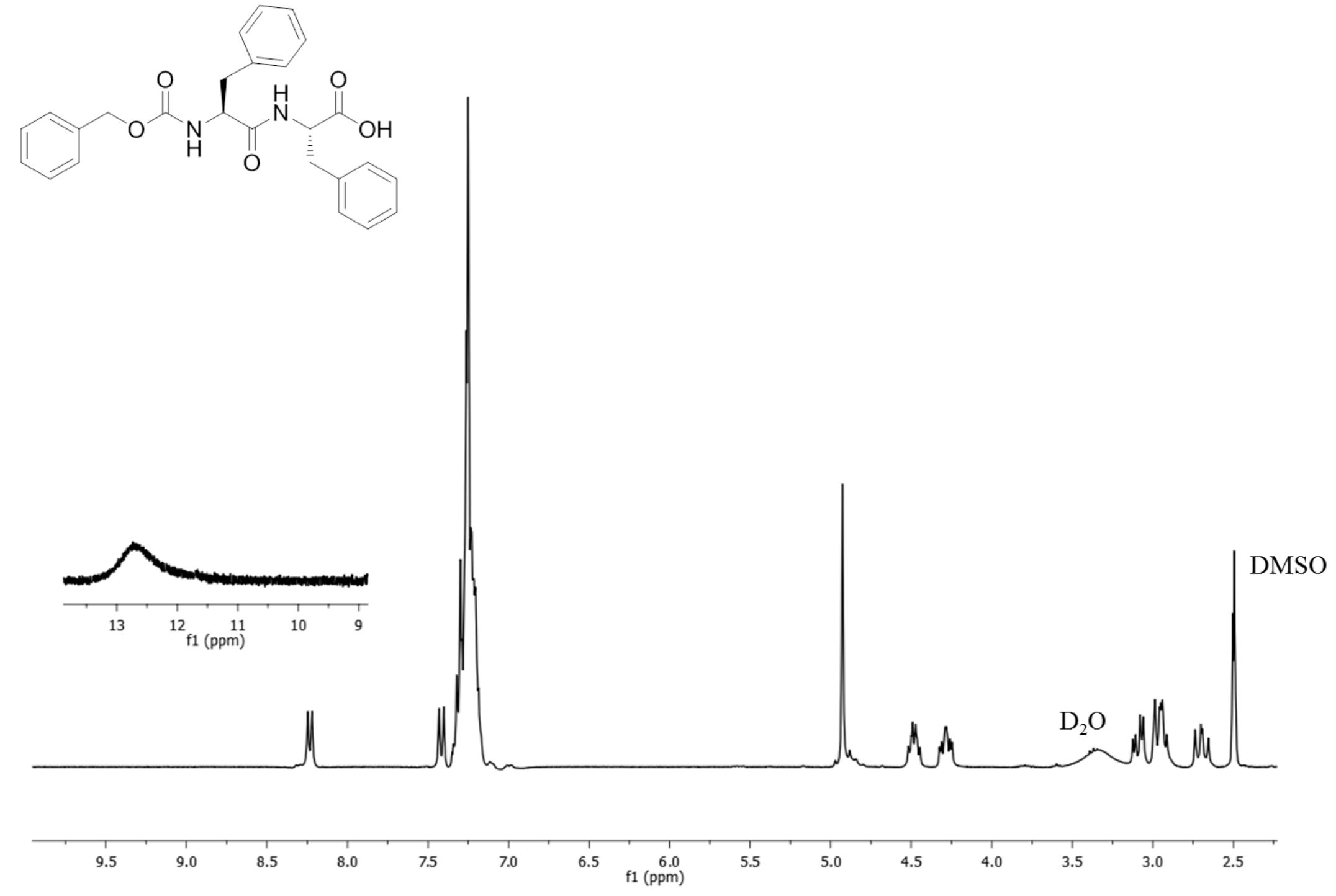




\section{ZPhePheOH}

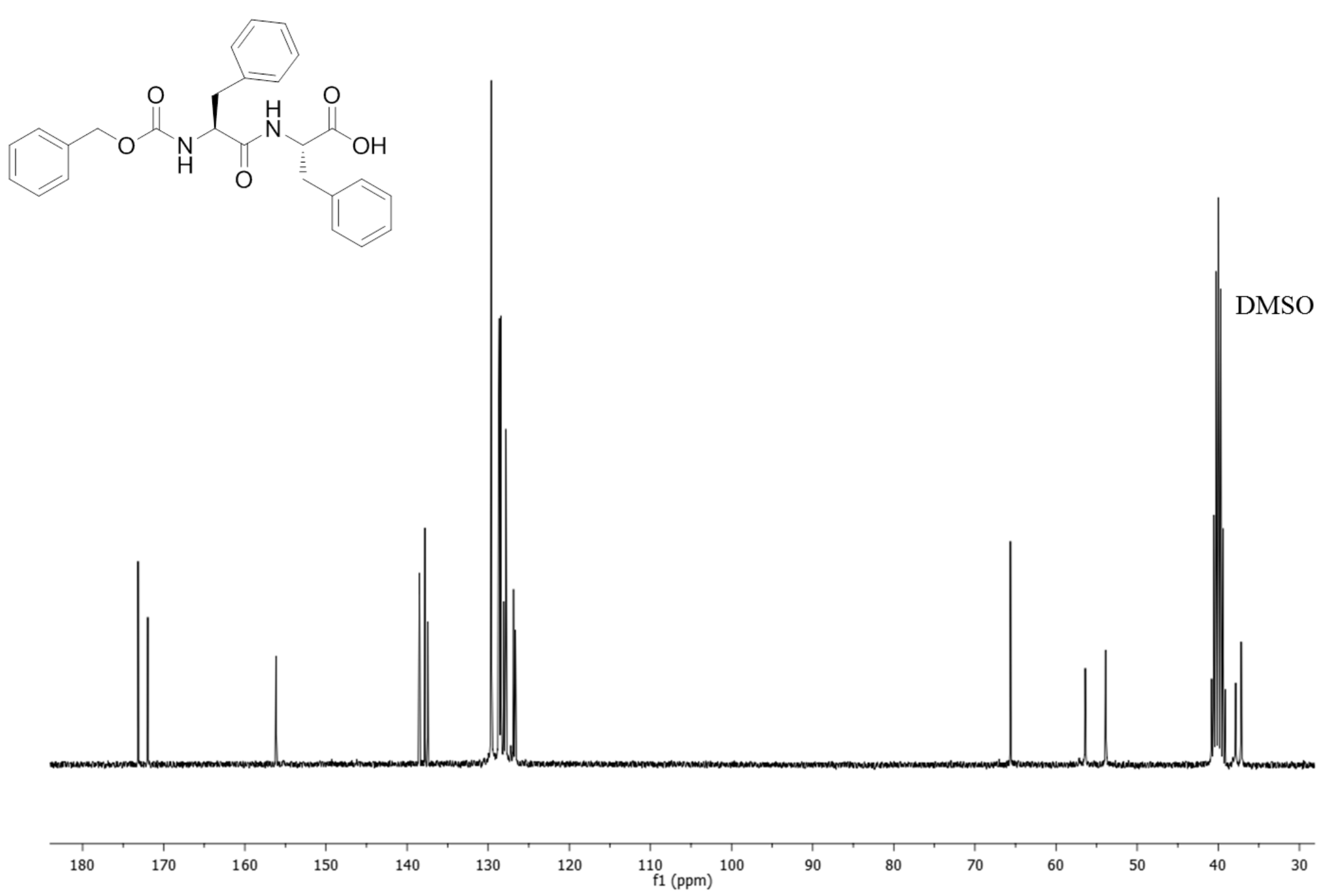




\section{ZAsp(tBu)PheOH}
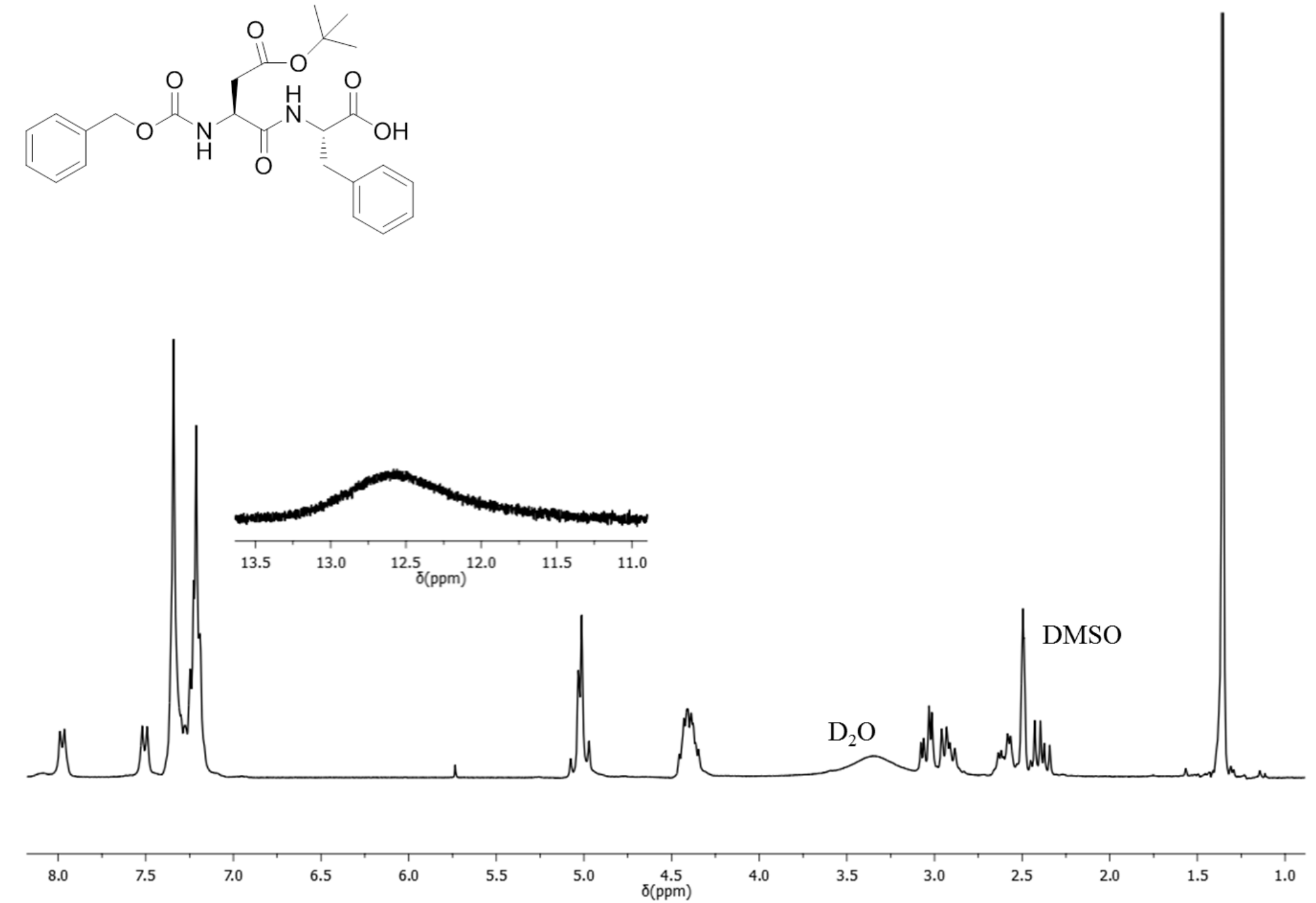


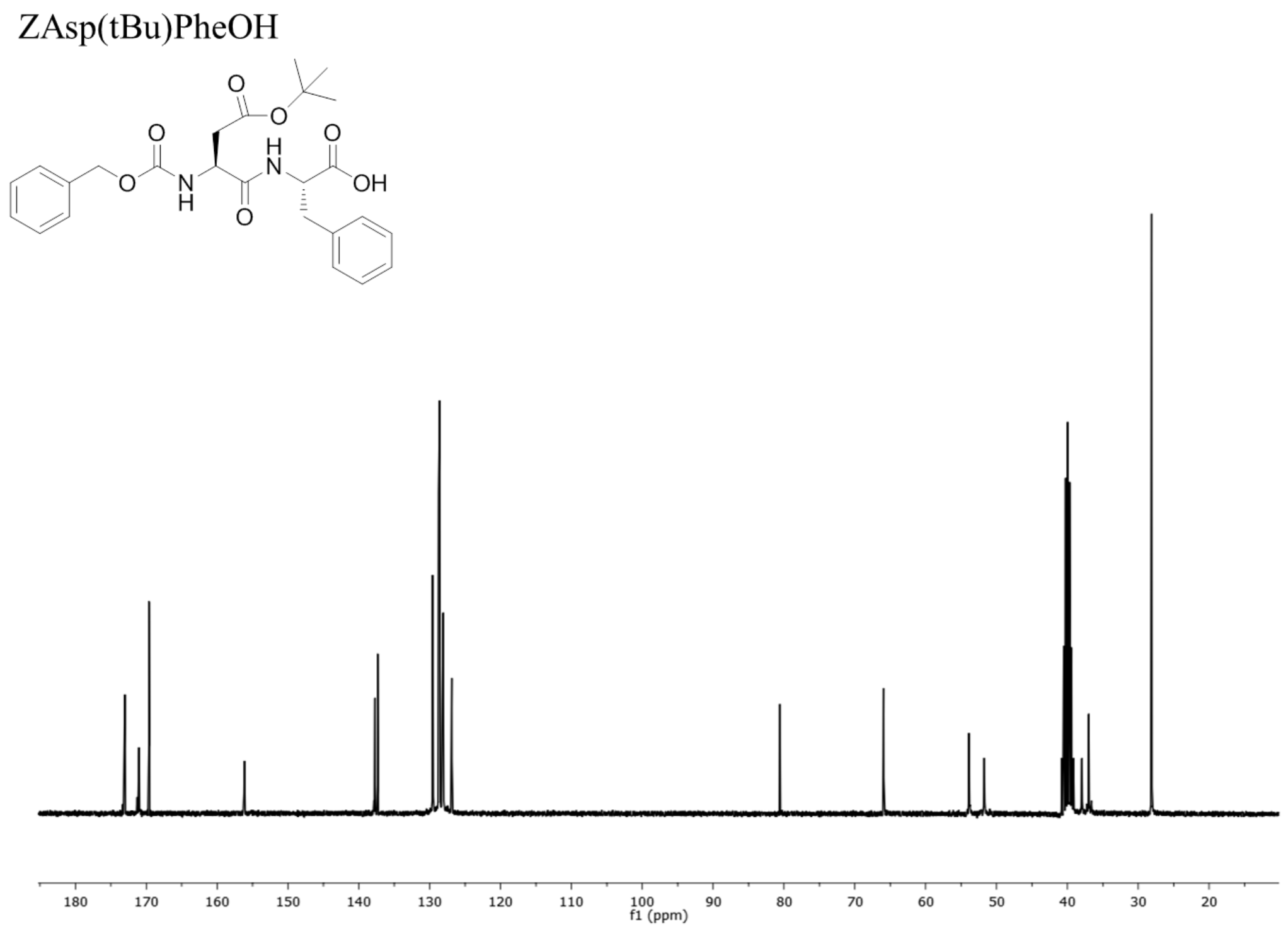




\section{ZPheAspAspPheNHPr}

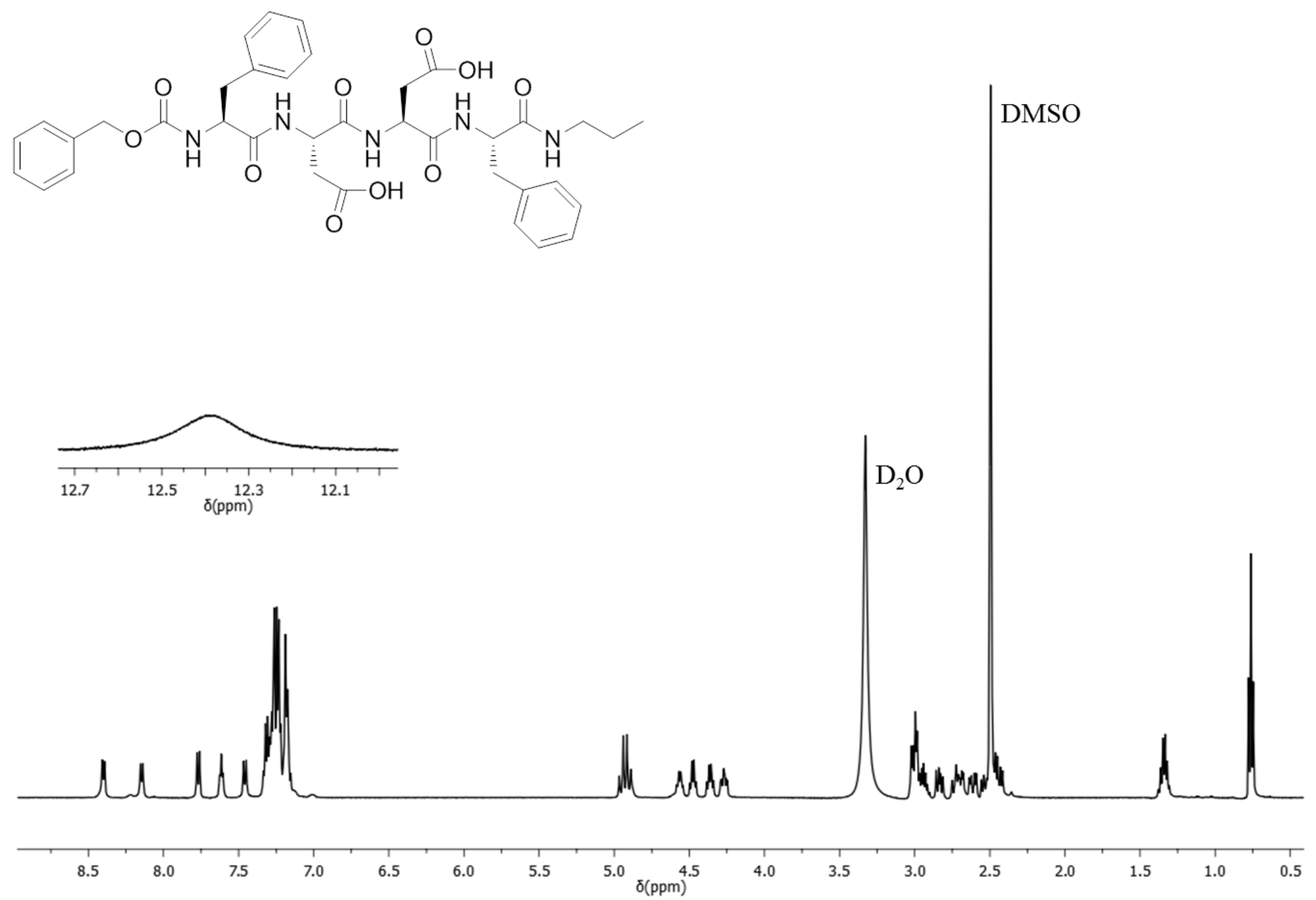




\section{ZPheAspAspPheNHPr}

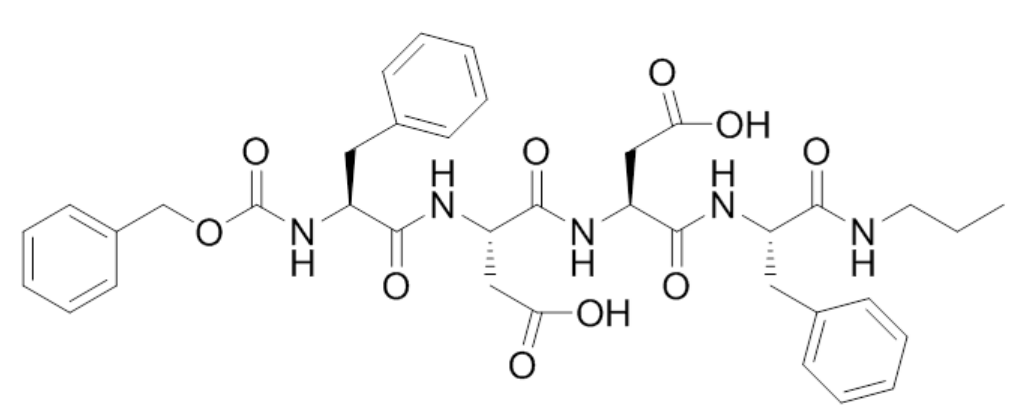

DMSO
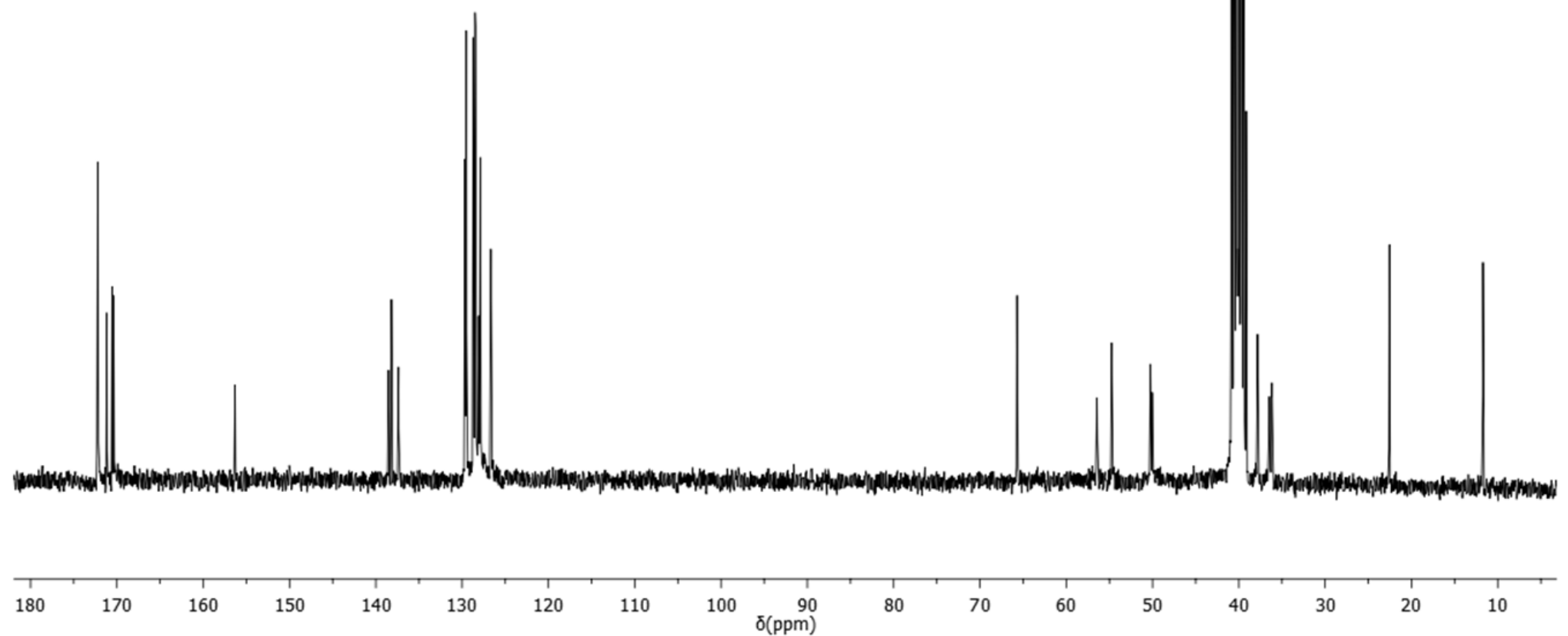


\section{ZAspPheAspPheNHPr}

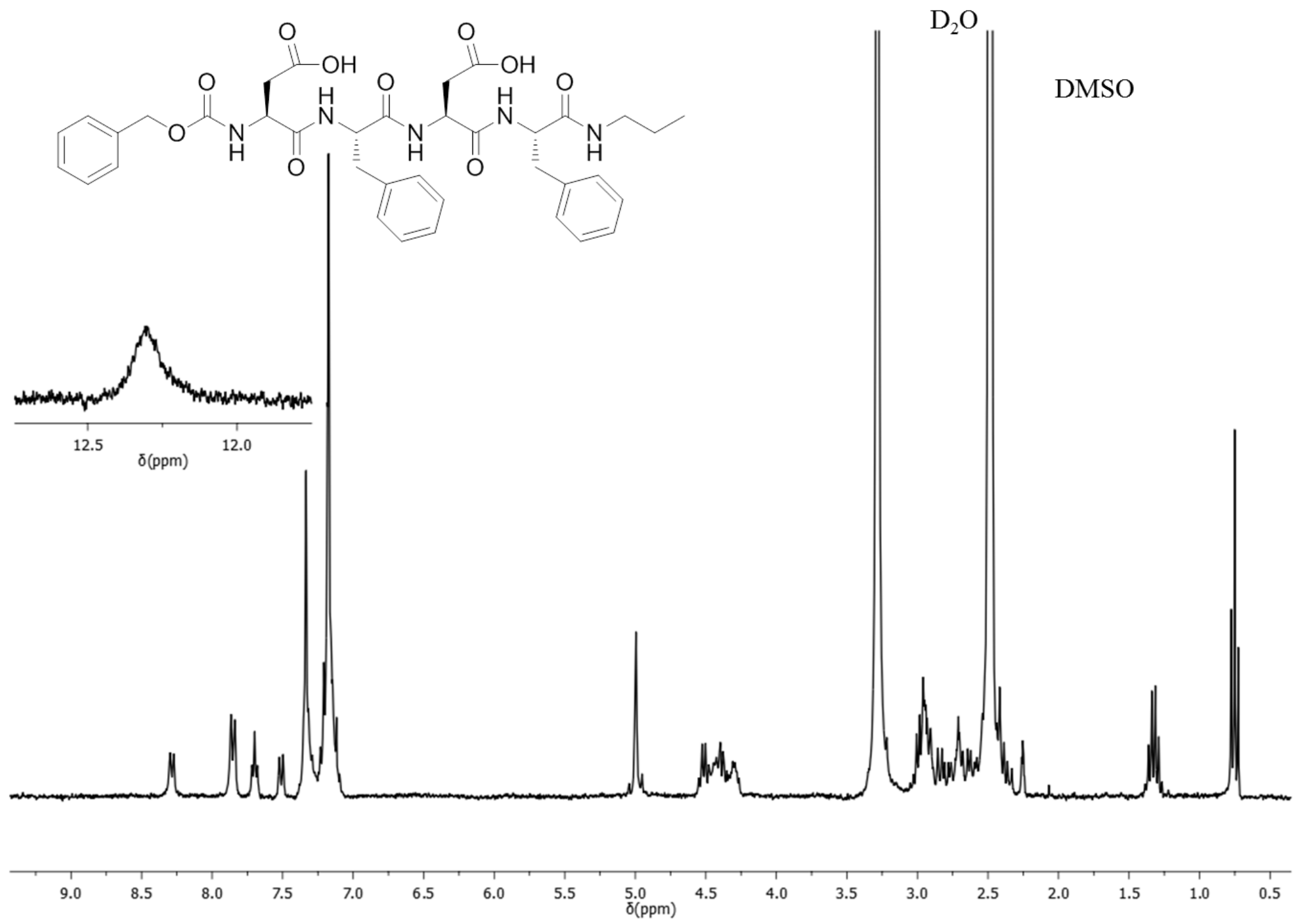




\section{ZAspPheAspPheNHPr}

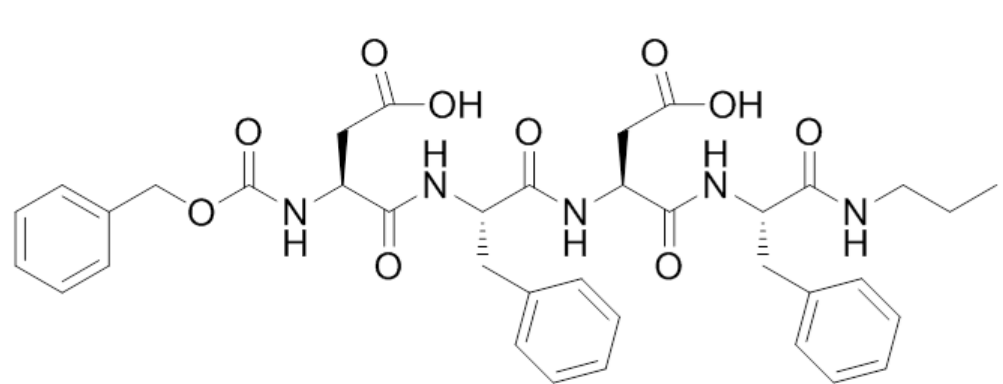

DMSO

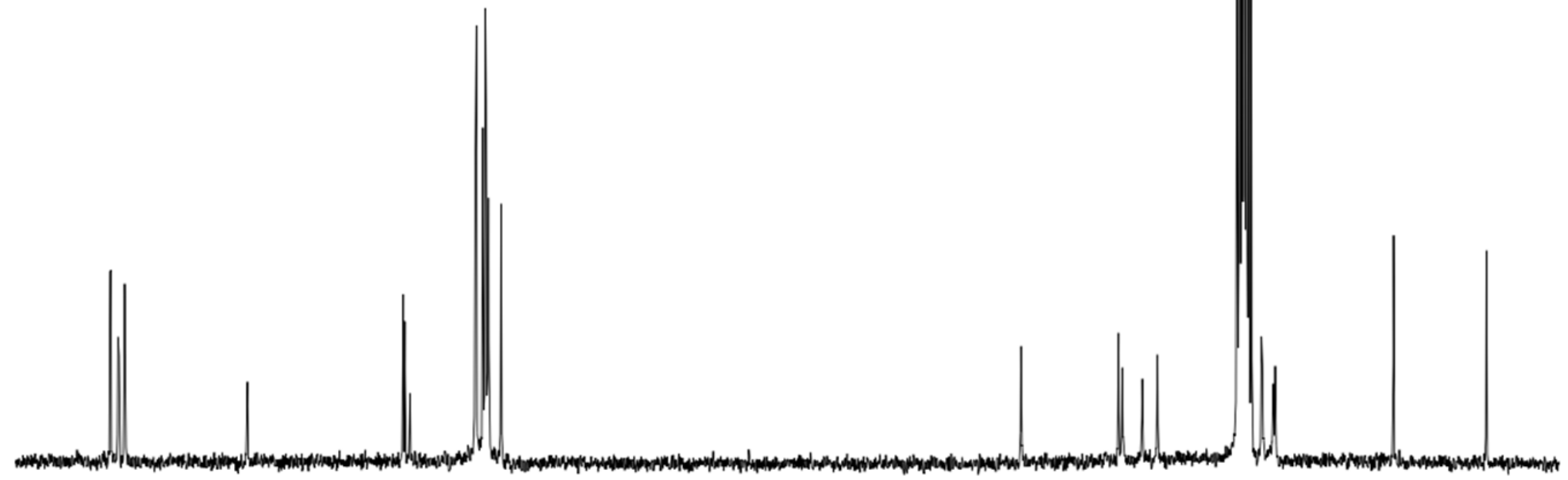

180

$160 \quad 150$

140

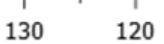

110

$\delta(\mathrm{ppm})$ 


\section{ZAspPhePheAspNHPr}

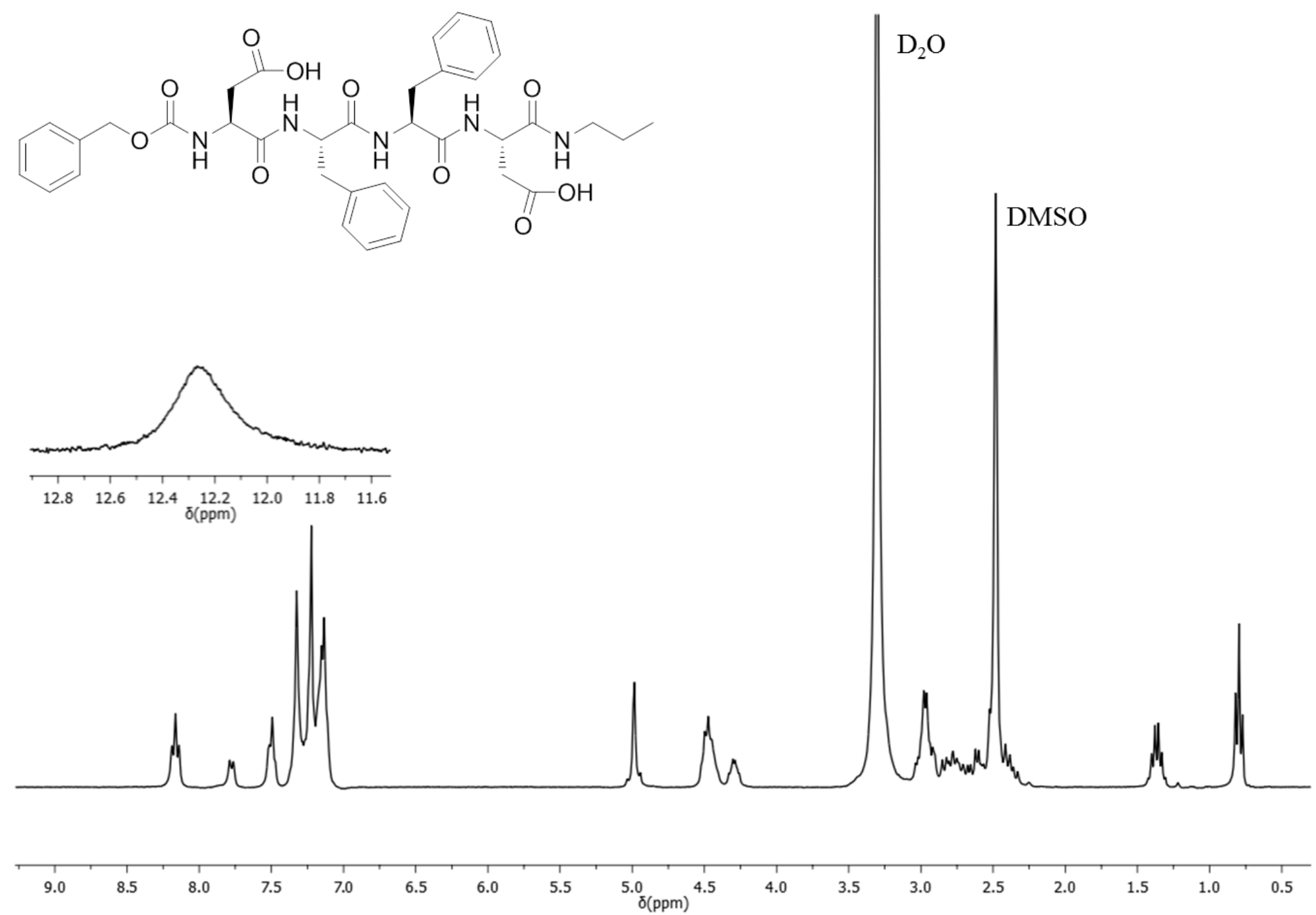


ZAspPhePheAspNHPr

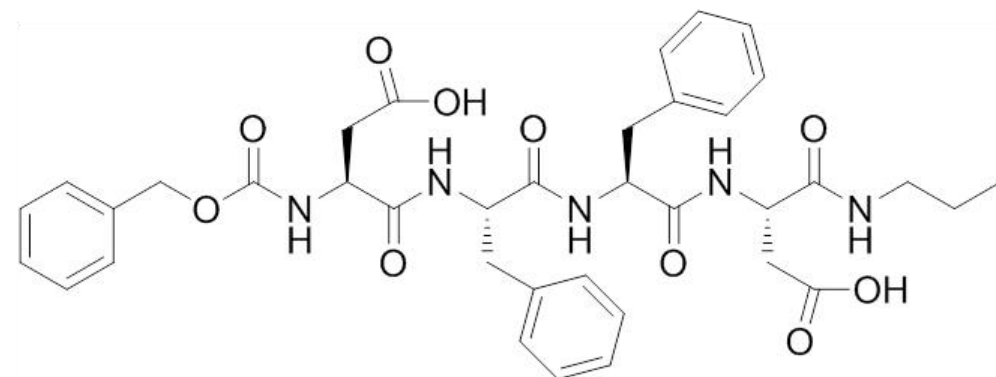

DMSO

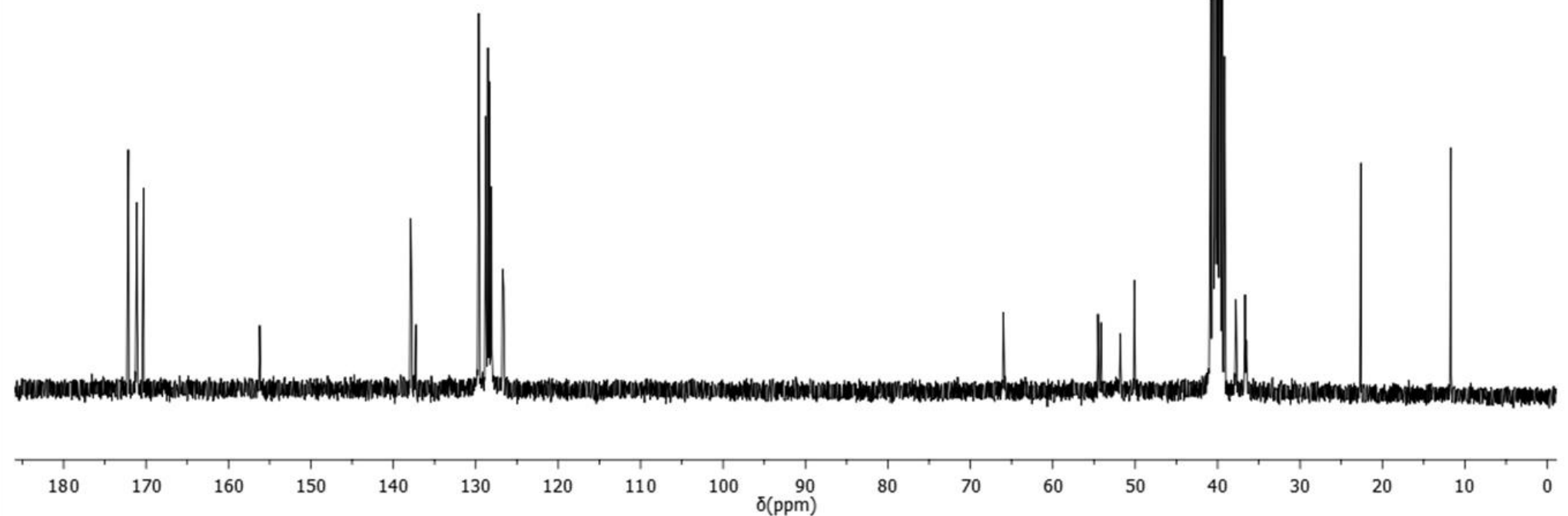




\section{ZGlyGlyOH}

$\overbrace{\mathrm{O}}^{\mathrm{IN}} \mathrm{O}_{\mathrm{O}}^{\mathrm{O}} \mathrm{OH}_{\mathrm{O}}^{\mathrm{O}}$

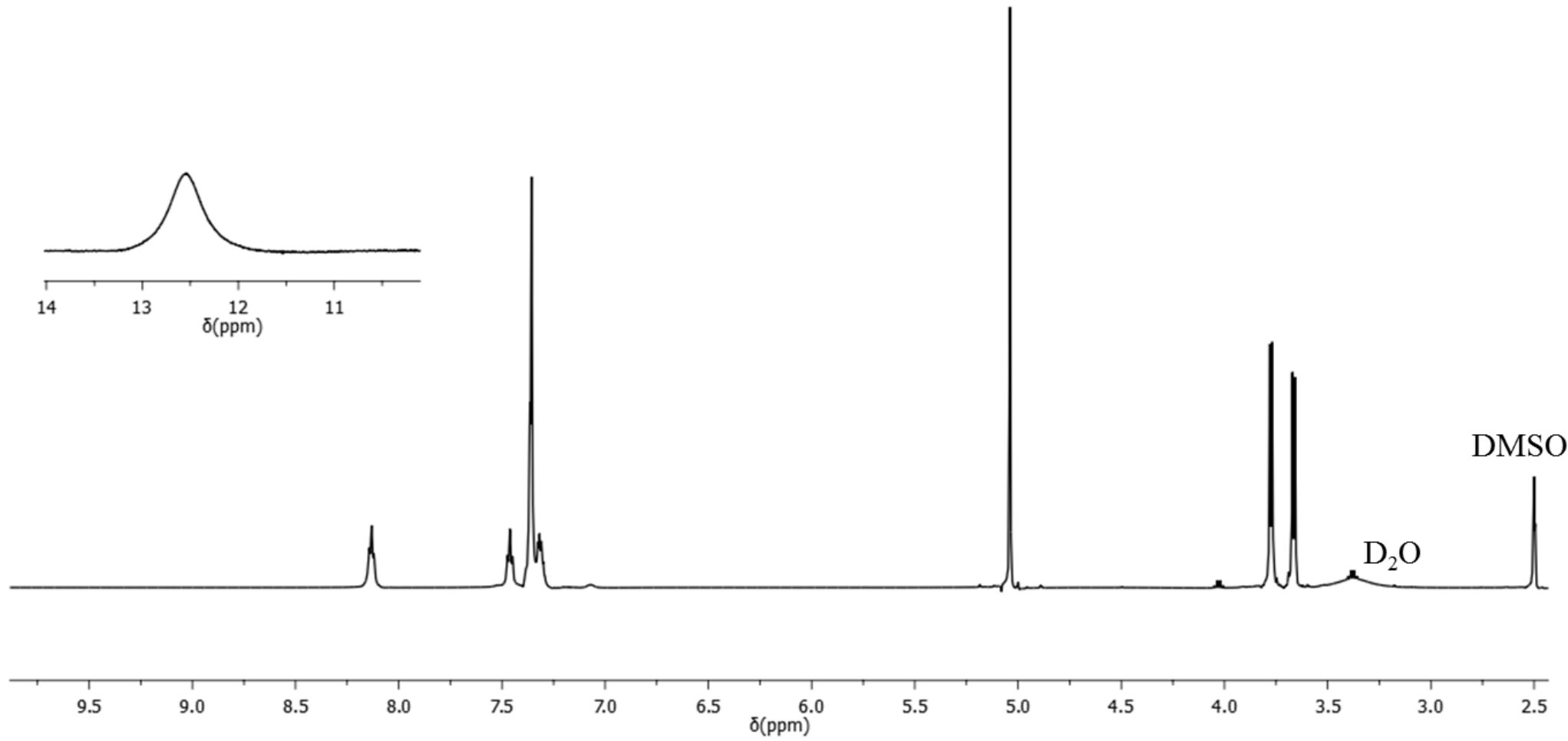




\section{ZGlyGlyOH}

$\overbrace{\mathrm{O}}^{\mathrm{N}} \mathrm{O}_{\mathrm{O}}^{\mathrm{O}} \mathrm{N}_{\mathrm{OH}}^{\mathrm{O}}$

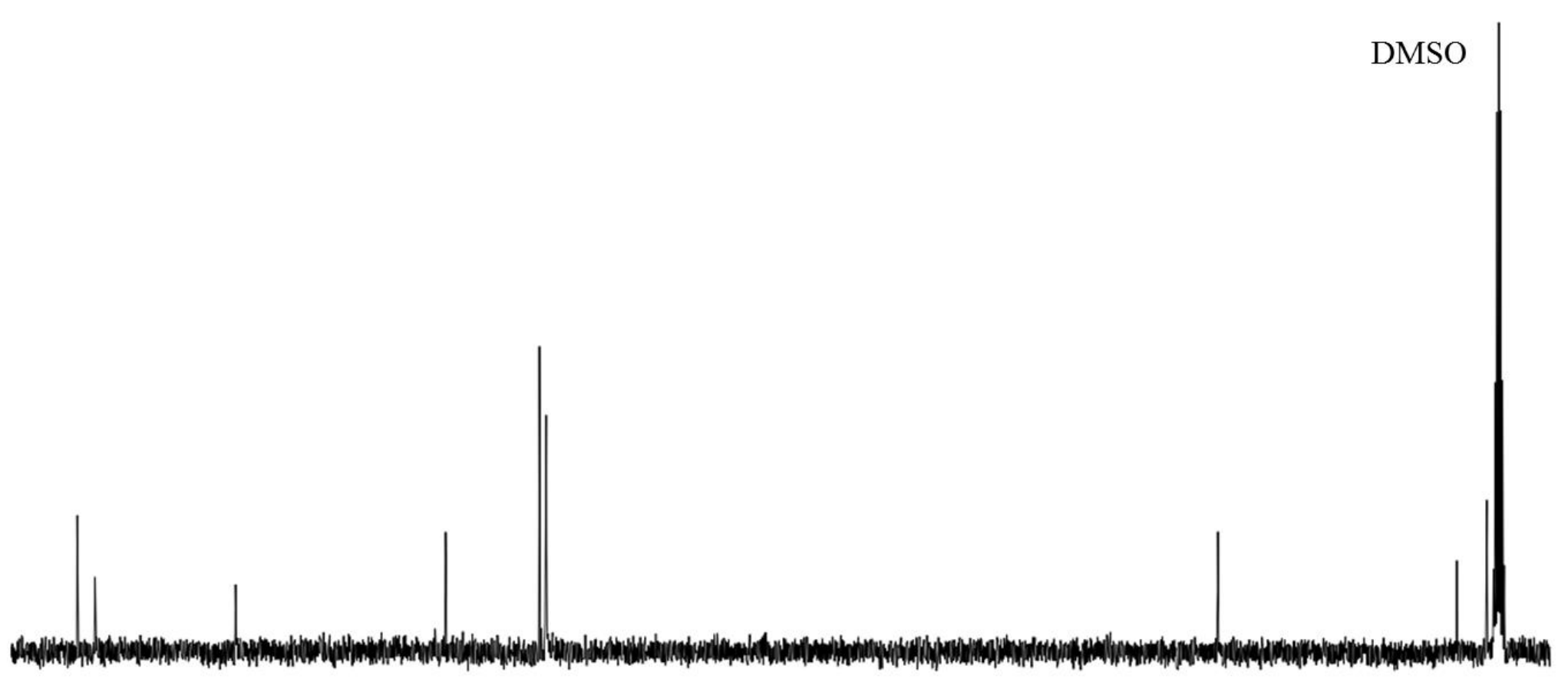

$\begin{array}{lllllllllllllllllllllllllllllllllll}175 & 170 & 165 & 160 & 155 & 150 & 145 & 140 & 135 & 130 & 125 & 120 & 115 & 110 & 105 & 100 & 95 & 90 & 85 & 80 & 75 & 70 & 65 & 60 & 55 & 50 & 45 & 40 & 35\end{array}$ 


\section{ZGlyGlyGlyOH}

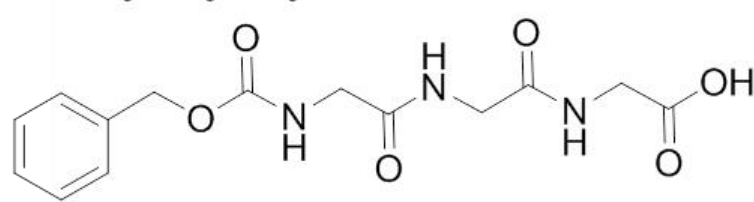

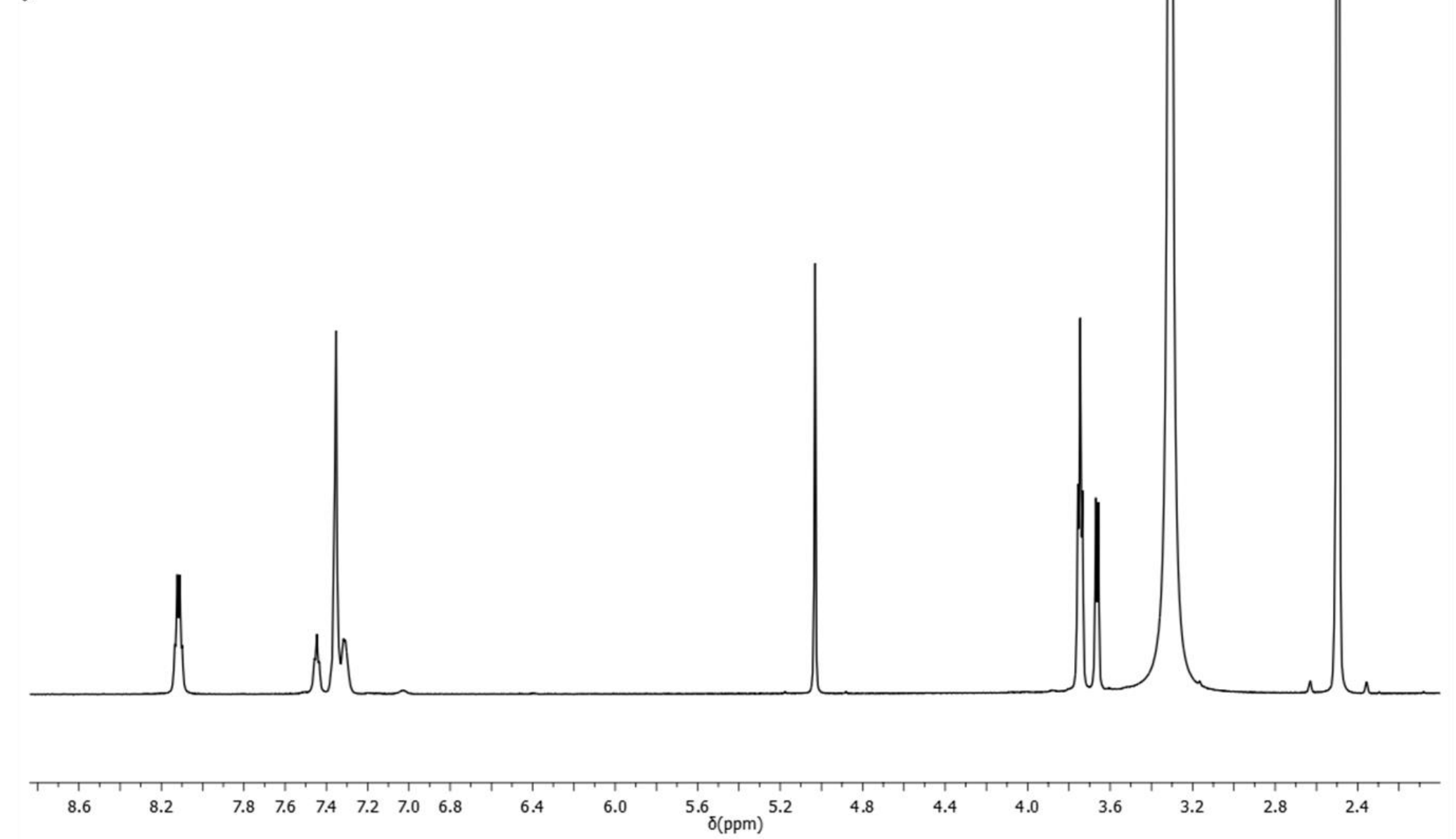




\section{ZGlyGlyGlyOH}

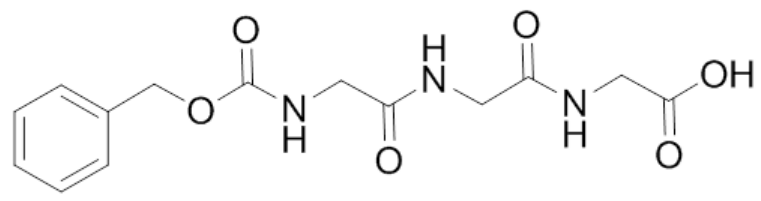

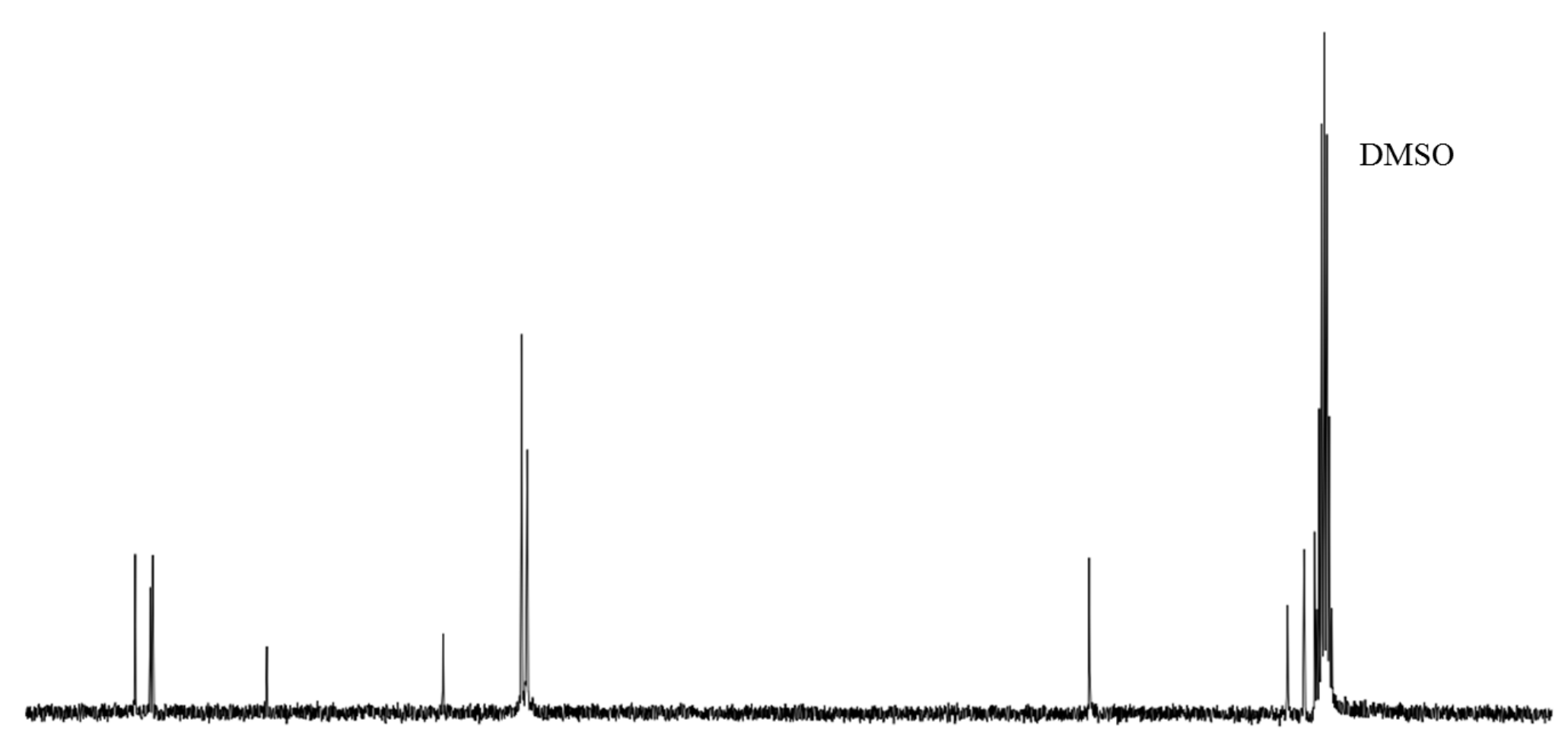

180

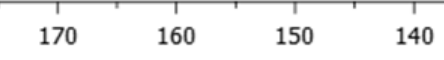

130

120

110

$\stackrel{100}{\delta(\mathrm{ppm})}$

90

80

70

60

50

40

30

20 


\section{PheNHPr}
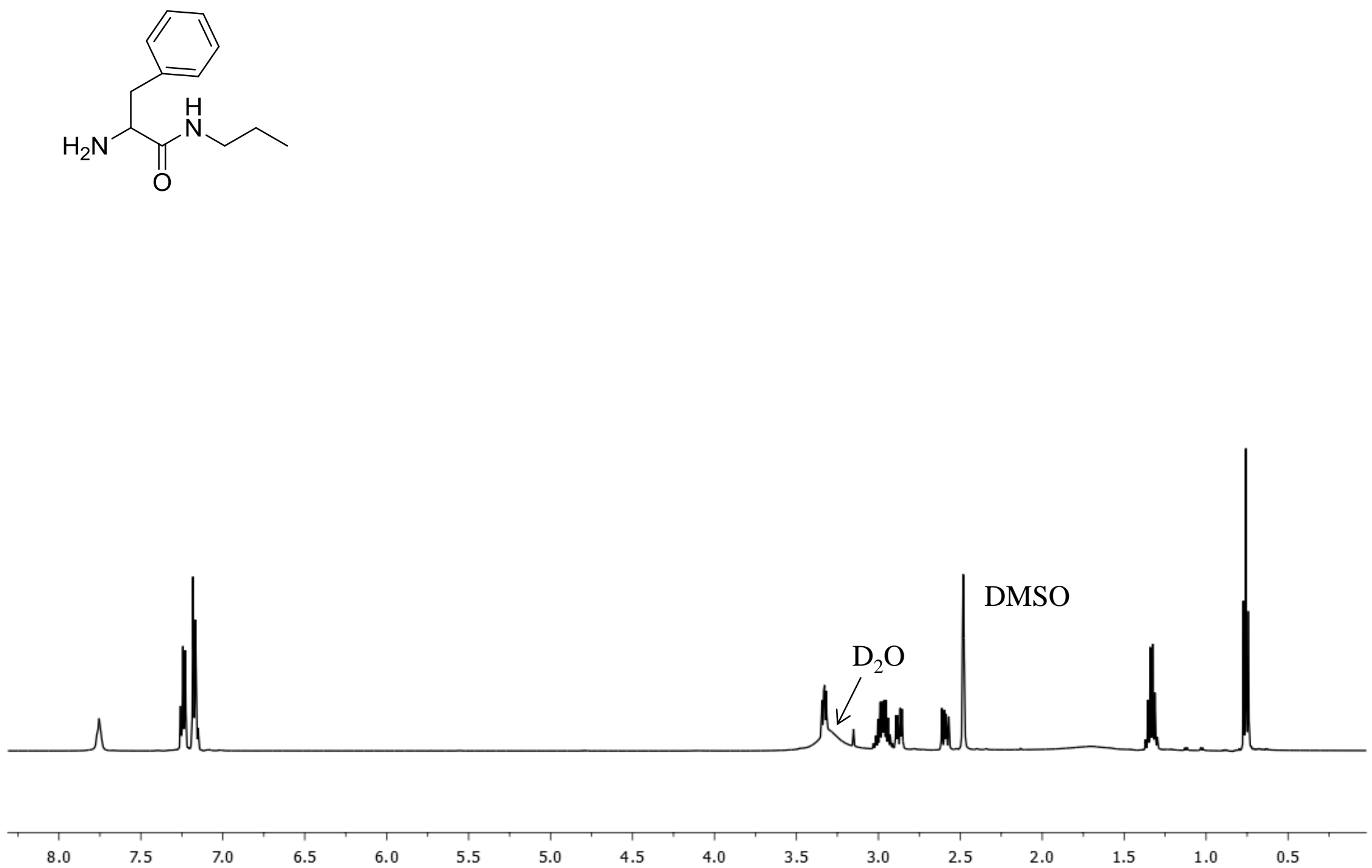


\section{PheNHPr}
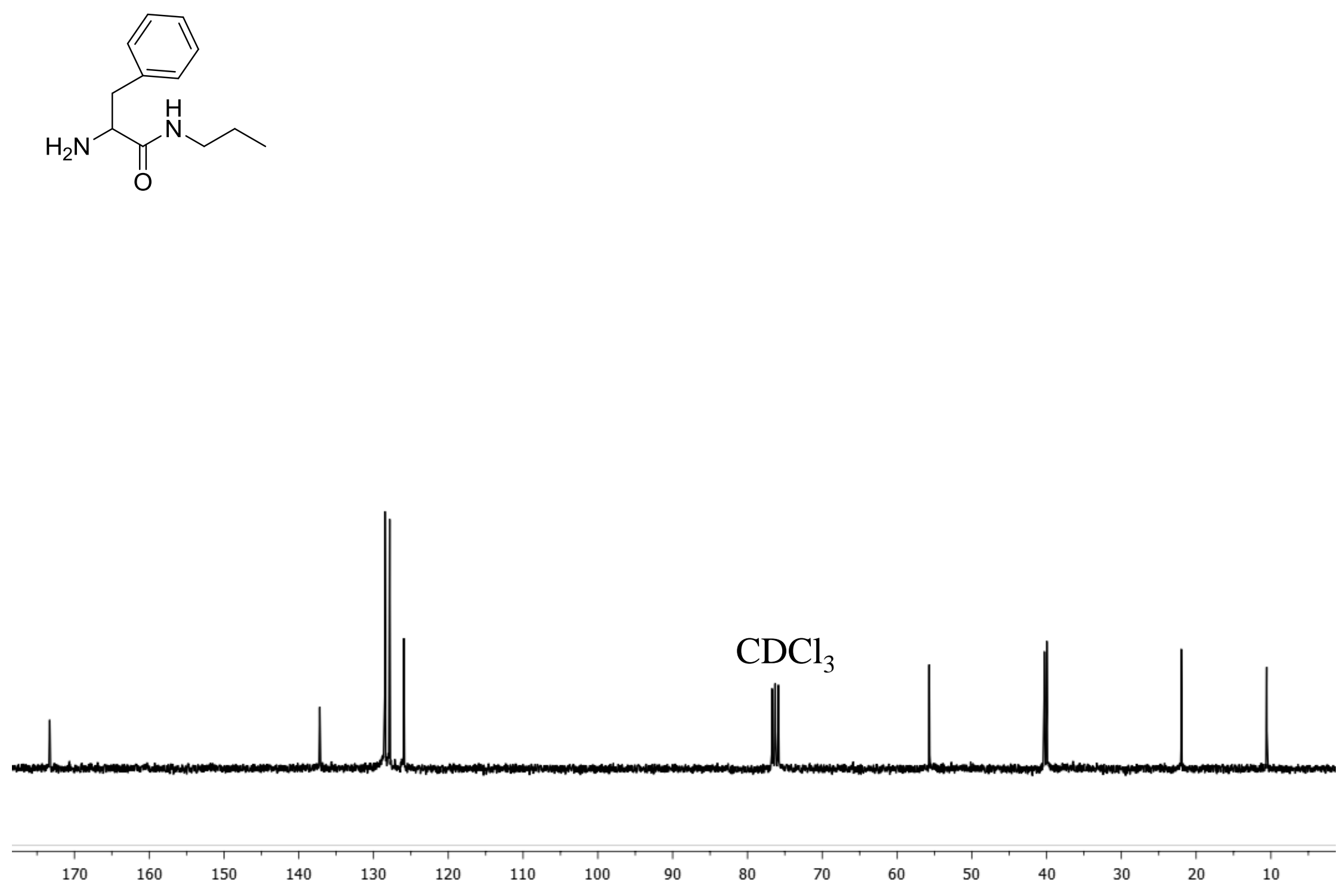